\title{
Operational approach in the weak-field measurement of polarization fluctuations
}

\author{
T. Hakioğlu \\ Physics Department, Bilkent University, 06533-Ankara, Turkey
}

(Received 1 July 1998)

\begin{abstract}
The operational approach to the measurement of phase studied by Noh, Fougerès, and Mandel [Phys. Rev. A 45, 424 (1992); 46, 2840 (1992); Phys. Rev. Lett. 71, 2579 (1993); Phys. Rev. A 47, 4535 (1993)] is applied to the measurement of the state of polarization of fully polarized light. Operational counterparts of the quantum Stokes parameters are introduced and their fluctuations are examined. It is shown that if the polarized field is weak, the measured fluctuations are influenced not only by the quantum properties of the source field but also that of the measurement. This character is reflected on the measured probability distributions of the parameters of polarization, which are also investigated independently for the fully polarized coherent states and the Fock states as the initial field strength is varied. Finally, connection between the operational approach to the measurement of polarization and the su(2) interferometry is examined. [S1050-2947(99)01402-X]

PACS number(s): 42.50.Dv, 85.60.Gz
\end{abstract}

\section{INTRODUCTION}

The idea of the operational approach as an experimental technique based on photon counting in the measurement of quantum-phase fluctuations was suggested in 1986 by Barnett and Pegg [1] in the context of a measured phase operator using certain homodyne experiments and more recently was formulated in detail by Noh, Fougères, and Mandel (NFM) [2]. The operational phase measurement is based on using $\mathrm{N}$-port quantum homodyne detectors of which the analogy with classical homodyne approach is based on the purpose of extracting information about the phase between two initial fields by performing a complete set of photocount measurements between the components of the field. This procedure of obtaining the phase information between two fields depends on the particular experimental scheme through its classical analogy of relating the relative photocount measurements to certain cosine and sine functions of the relative phase. Since, through this suggested analogy, different quantum measurement schemes would correspond to different classical ones, the information extracted for the relative phase is expected to be different for different experimental schemes. Indeed, this point has been demonstrated in the formulation of the operational phase measurement by NFM by starting with two different classical and quantum measurement schemes where one measurement used two-port homodyne detection whereas the second one used four-port homodyne detection [2,3]. The two-port measurement yields either the cosine or the sine information about the phase failing to give the full phase information. In the four-port scheme the simultaneous measurements were made possible by well-defined trigonometric operators of the relative phase where the full information on the phase and its fluctuations can be extracted. On the other hand, a comparison of NFM's operational approach with the operational approach introduced by Vogel and Schleich [4] has been compared by Lynch [5], who found agreement between the two operational schemes.

Another particularly important part of this scheme dependence manifests itself in the weak-field measurements in which the quantized nature of light as well as that of the detectors becomes crucial when the homodyne detectors have a relatively high probability of registering a few or null photocounts within the measurement time interval $T$. This being the case for a single set of measurements, one considers an ensemble of repeated measurements under the same initial conditions. There, each repeated measurement would have generally different but equally acceptable configurations of detected photons and one has to make a distinction between the outcome of a single measurement from the average outcome of a collection of such repeated measurements under otherwise the same conditions. Despite the fact that the experimental verification of NFM's operational approach was successfully made by the same group [6], the appearance of the discrete outcomes in the phase measurements in their scheme was subject to long and heavy discussions [7-10]. In this work we suggest another application of their approach to the operational measurement of the state of polarization of a fully polarized source. In an earlier publication [11] we investigated a particular extension of NFM's operational approach to the measurement of the Stokes parameters of a fully polarized weak coherent light. In this work we will extend this formalism introduced in [11] to a more general framework by including the calculations for the measured probability distributions of the polarization fluctuations and also examine the case thoroughly when the initial field is a fully polarized Fock state.

We start with a brief outline of the operational approach to the measurement of polarization fluctuations when the polarized field is given in a classical as well as a quantum state. In Sec. II we present the general formalism of calculating the polarization fluctuations and their corresponding probability distributions. Sections II A and II B are devoted to the specific calculations corresponding to two different fully polarized initial quantum states of the field as coherent and Fock states, respectively. Section II C is devoted to the connections between the operational approach and the su(2) interferometry.

Classically, the state of polarization of a fully polarized monochromatic field $E_{i}=\epsilon_{i} \cos \left(\omega t+\delta_{i}\right)$, where $i=1,2$ are the polarization indices of two preselected orthogonal polariza tion eigenmodes, can be manifestly described by four Stokes 


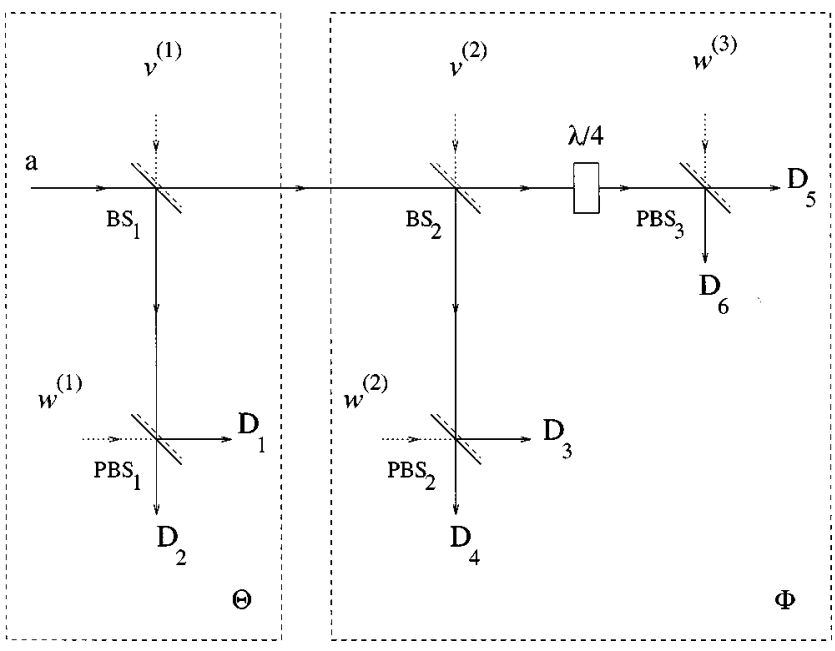

FIG. 1. Experimental setup to measure the classical and quantum Stokes parameters. Note that this setup is also able to measure a total of six Stokes parameters for partially polarized light.

parameters $s_{m}(m=0,1,2,3)$ as $[12-15]$

$$
\begin{gathered}
s_{0}=\frac{1}{2}\left(\left\langle E_{1}^{2}\right\rangle+\left\langle E_{2}^{2}\right\rangle\right), \\
s_{1}=\frac{1}{2}\left(\left\langle E_{1}^{2}\right\rangle-\left\langle E_{2}^{2}\right\rangle\right), \\
s_{2}=\left(\left\langle E_{1}^{2}\right\rangle\left\langle E_{2}^{2}\right\rangle\right)^{1 / 2} \cos \phi, \\
s_{3}=\left(\left\langle E_{1}^{2}\right\rangle\left\langle E_{2}^{2}\right\rangle\right)^{1 / 2} \sin \phi,
\end{gathered}
$$

where $\phi=\delta_{2}-\delta_{1}$ is the optical (temporal) phase and $I_{i}$ $=\left\langle E_{i}^{2}\right\rangle$ is the intensity of the corresponding $i$ th component $(i=1,2)$. We now describe an experimental setup based on a set of photocount measurements for the purpose of investigating the fluctuations in the measurement of the classical Stokes parameters in Eqs. (1) and their corresponding quantum counterparts.

\section{A. Classical measurement scheme}

Within the operational approach, it is possible to measure all classical Stokes parameters in terms of the various components of the intensity. The experimental scheme is shown in Fig. 1. The initial field enters the setup through the $50 \%$ $50 \%$ beam splitter $\mathrm{BS}_{1}$. One of the output beams of $\mathrm{BS}_{1}$ is sent to a polarizing beam splitter $\mathrm{PBS}_{1}$, which defines a reference frame 1,2 for the relative angular orientation of all other polarizing beam splitters. The other arm of the beam leaving $\mathrm{BS}_{1}$ is sent to $\mathrm{BS}_{2}$ as an input, leading to the second part of the experiment where the simultaneous measurements of $\cos \phi$ and $\sin \phi$ are realized independently from the first part. $\mathrm{PBS}_{2}$ is aligned at a $45^{\circ}$ angle with respect to the reference frame selected by $\mathrm{PBS}_{1}$. The intensities measured at the detectors $D_{3}$ and $D_{4}$ yield the measured values of $\cos \phi$ and its moments. For the $\sin \phi$ measurement, the phase of the remaining arm of the field is shifted by $\pi / 2$ via a quarter wave plate $\lambda / 4$. The field is then sent to $\mathrm{PBS}_{3}$, which is

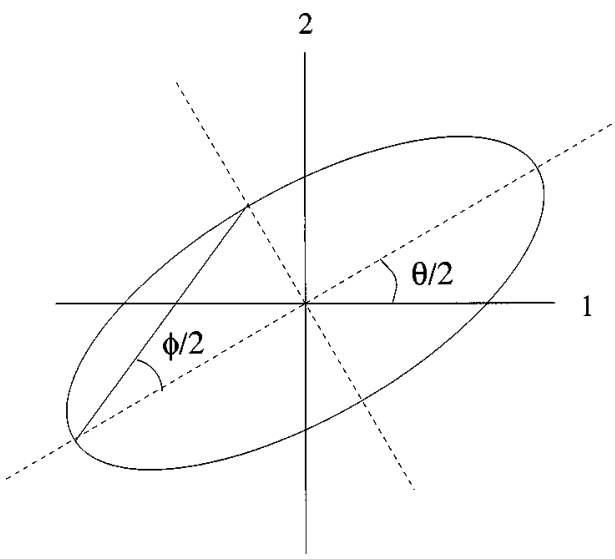

FIG. 2. Ellipsometry for the fully polarized transverse electric field $\mathbf{E}$ in the tangent plane. Angular parameters are shown as defined in Eqs. (4).

aligned in parallel to $\mathrm{PBS}_{2}$. A simple calculation shows that the classical intensities measured at all detectors $D_{i}(i$ $=1, \ldots, 6)$ are given by

$$
\begin{aligned}
& I_{1}=\frac{1}{2}\left\langle E_{1}^{2}\right\rangle, \\
& I_{2}=\frac{1}{2}\left\langle E_{2}^{2}\right\rangle,
\end{aligned}
$$

$$
\begin{aligned}
& I_{3}=\frac{1}{4}\left[\left\langle E_{1}^{2}\right\rangle+\left\langle E_{2}^{2}\right\rangle+2 \sqrt{\left\langle E_{1}^{2}\right\rangle\left\langle E_{2}^{2}\right\rangle} \cos \phi\right], \\
& I_{4}=\frac{1}{4}\left[\left\langle E_{1}^{2}\right\rangle+\left\langle E_{2}^{2}\right\rangle-2 \sqrt{\left\langle E_{1}^{2}\right\rangle\left\langle E_{2}^{2}\right\rangle} \cos \phi\right], \\
& I_{5}=\frac{1}{4}\left[\left\langle E_{1}^{2}\right\rangle+\left\langle E_{2}^{2}\right\rangle+2 \sqrt{\left\langle E_{1}^{2}\right\rangle\left\langle E_{2}^{2}\right\rangle} \sin \phi\right], \\
& I_{6}=\frac{1}{4}\left[\left\langle E_{1}^{2}\right\rangle+\left\langle E_{2}^{2}\right\rangle-2 \sqrt{\left\langle E_{1}^{2}\right\rangle\left\langle E_{2}^{2}\right\rangle} \sin \phi\right] .
\end{aligned}
$$

Equations (1) and (2) imply that the classical Stokes parameters can be extracted operationally by measuring all field intensities $I_{i}(i=1, \ldots, 6)$. In terms of these intensities, the Stokes parameters are simply given by

$$
\begin{aligned}
& s_{0}=\left(I_{1}+I_{2}\right), \quad s_{1}=\left(I_{1}-I_{2}\right), \\
& s_{2}=\left(I_{3}-I_{4}\right), \quad s_{3}=\left(I_{5}-I_{6}\right) .
\end{aligned}
$$

In [11] we parametrized the polarized field in terms of the functions

$$
\cos \theta=s_{1} / s_{0}, \quad \sin \theta=\sqrt{s_{0}^{2}-s_{1}^{2}} / s_{0},
$$

$$
\cos \phi=s_{2} / \sqrt{s_{2}^{2}+s_{3}^{2}}, \quad \sin \phi=s_{3} / \sqrt{s_{2}^{2}+s_{3}^{2}} .
$$

This particular choice of parameters proves to be very convenient in the quantum operational measurements on fully polarized light. They also lead us naturally to Poincaré's geometric interpretation of polarization [12-15]. Here $\theta$ and $\phi$ are physical parameters as shown in Fig. 2. Their 
values are directly connected with the ellipsometry of the polarized field. From now on we will adopt this parametrization and study the polarization fluctuations in terms of the fluctuations of these observables.

\section{B. Quantum measurement scheme}

The classical description above is adequate when the field intensity is sufficiently high. The vacuum fields, which are not present in the classical approach, are necessary for the correct quantum description of the apparatus as well as the field observables.

In Fig. 1 the field operators $\hat{d}_{1}, \hat{d}_{2}$ at the output of $\mathrm{PBS}_{1}$ are related to the input field components $\hat{a}_{1}, \hat{a}_{2}$ as [11]

$$
\hat{d}_{1}=\frac{1}{\sqrt{2}}\left(r \hat{a}_{1}+t \hat{v}_{1}^{(1)}\right), \quad \hat{d}_{2}=\frac{1}{\sqrt{2}}\left(r \hat{a}_{2}+t \hat{v}_{2}^{(1)}\right),
$$

where $r=i / \sqrt{2}$ and $t=1 / \sqrt{2}$ are the field reflection and transmission coefficients and $\hat{v}_{j}^{(1)}(j=1,2)$ are the polarized vacuum fields entering through the vacuum port of $\mathrm{BS}_{1}$. If the measurement scheme in Fig. 1 is extended to include the photodetectors $D_{i}(i=3,4,5,6)$, then $\phi$ measurements can be made compatible with a proper quantum treatment of all fields. The output fields of $\mathrm{PBS}_{2}$ and $\mathrm{PBS}_{3}$ at $D_{3}, D_{4}, D_{5}, D_{6}$ are given by

$$
\begin{aligned}
& \hat{d}_{3}=\frac{1}{\sqrt{2}}\left[\left(t r \hat{a}_{1}+r^{2} \hat{v}_{1}^{(1)}+t \hat{v}_{1}^{(2)}\right)+\left(t r \hat{a}_{2}+r^{2} \hat{v}_{2}^{(1)}+t \hat{v}_{2}^{(2)}\right)\right], \\
& \hat{d}_{4}=\frac{1}{\sqrt{2}}\left[-\left(t r \hat{a}_{1}+r^{2} \hat{v}_{1}^{(1)}+t \hat{v}_{1}^{(2)}\right)+\left(t r \hat{a}_{2}+r^{2} \hat{v}_{2}^{(1)}+t \hat{v}_{2}^{(2)}\right)\right],
\end{aligned}
$$

$$
\hat{d}_{5}=\frac{1}{\sqrt{2}}\left[i\left(t^{2} \hat{a}_{1}+t r \hat{v}_{1}^{(1)}+r \hat{v}_{1}^{(2)}\right)+\left(t^{2} \hat{a}_{2}+t r \hat{v}_{2}^{(1)}+r \hat{v}_{2}^{(2)}\right)\right]
$$

$\hat{d}_{6}=\frac{1}{\sqrt{2}}\left[-i\left(t^{2} \hat{a}_{1}+t r \hat{v}_{1}^{(1)}+r \hat{v}_{1}^{(2)}\right)+\left(t^{2} \hat{a}_{2}+t r \hat{v}_{2}^{(1)}+r \hat{v}_{2}^{(2)}\right)\right]$.

In connection with their classical counterparts in Eqs. (3), we are now at a point to suggest the quantum Stokes parameters for the field operators $\hat{d}_{i}$ within this operational approach in terms of the observable photon number operators $\hat{n}_{i}=\hat{d}_{i}^{\dagger} \hat{d}_{i}$ as

$$
\begin{array}{ll}
\hat{\Sigma}_{0}=\hat{n}_{1}+\hat{n}_{2}, & \hat{\Sigma}_{1}=\hat{n}_{1}-\hat{n}_{2}, \\
\hat{\Sigma}_{2}=\hat{n}_{3}-\hat{n}_{4}, & \hat{\Sigma}_{3}=\hat{n}_{5}-\hat{n}_{6} .
\end{array}
$$

In Eqs. (6) all field operators commute as a manifestation of the vacuum fields. Hence, in Eqs. (7) we have $\left[\hat{\Sigma}_{i}, \hat{\Sigma}_{j}\right]=0$ $(i \neq j)$ and all photon number operators can be simultaneously measured at the detectors $D_{i}(i=1, \ldots, 6)$. As a result, Eqs. (7) are compatible with their classical counterparts in Eqs. (3). This property of the $\hat{\Sigma}_{i}(i=0, \ldots, 3)$ operators allows us to further suggest an extension (4) to their operator counterparts as

$$
\begin{gathered}
\hat{C}_{\theta}=\hat{\Sigma}_{0}^{-1} \hat{\Sigma}_{1}, \quad \hat{S}_{\theta}=\left(1-\hat{C}_{\theta}^{2}\right)^{1 / 2} \\
\hat{C}_{\phi}=\hat{\Sigma}_{2}\left(\hat{\Sigma}_{2}^{2}+\hat{\Sigma}_{3}^{2}\right)^{-1 / 2}, \quad \hat{S}_{\phi}=\hat{\Sigma}_{3}\left(\hat{\Sigma}_{2}^{2}+\hat{\Sigma}_{3}^{2}\right)^{-1 / 2} .
\end{gathered}
$$

$\hat{C}_{\theta}, \hat{S}_{\theta}$ and $\hat{C}_{\phi}, \hat{S}_{\phi}$ are well-defined and compatible quantum observables. They commute with each other and satisfy the operator relations $\hat{C}_{\theta}^{2}+\hat{S}_{\theta}^{2}=1$ and $\hat{C}_{\phi}^{2}+\hat{S}_{\phi}^{2}=1$ and, as a result, can be measured simultaneously.

One of the benefits of adopting Eqs. (7) and (8) is that all measurements are now based on pure photon counting depending on the measured photocounts at the detectors $D_{i}(i$ $=1, \ldots, 6)$ and hence they do not involve any temporal interference effects. This is an advantage of the operational measurement, which will be transparent later in our discussion of the weak-field limit.

Equations (8), hereinafter referred to as the operational quantum Stokes parameters (OQSP), are the most convenient choice for $\hat{\Sigma}_{i}(i=0, \ldots, 3)$ befitting the purpose of the photocount measurement scheme of Fig. 1. All operators in Eqs. (8) are now compatible with the classical variables of Eqs. (4) as long as the measurements of the $\hat{\Sigma}_{2}$ and $\hat{\Sigma}_{3}$ operators do not yield zero simultaneously.

\section{MEASUREMENT OF POLARIZATION FLUCTUATIONS IN WEAK FIELDS}

The operational approach as applied to the polarization measurement of a fully polarized and weak initial field is based on individual detections of single photons where the quantum nature of the field as well as that of the detection mechanisms is dominant. The influence of the direct quantum homodyne detection on the statistics of a quantum measurement has been examined by Mandel [16], Kelley and Kleiner [17], and Glauber [18] and expressed in the form of a combined quantum probability distribution

$$
\mathcal{P}\left(\left\{n_{j}\right\}\right)=\prod_{j=1}^{N}:\left(\hat{d}_{j}^{\dagger} \hat{d}_{j}\right)^{n_{j}} \exp \left(-\hat{d}_{j}^{\dagger} \hat{d}_{j}\right) / n_{j} !
$$

where : : accounts for the normal ordering of the operators $\hat{d}_{i}^{\dagger}, \hat{d}_{i}$ inside and $\hat{d}_{i}^{\dagger} \hat{d}_{i}$ corresponds to the photon number operator. Throughout the calculations the measurement time interval will be assumed to be much smaller than the coherence time (which is naturally satisfied for a monochromatic field) and much larger than the inverse of the oscillation frequency of the field. Under these conditions it is possible to consider the simplest case when the photocount measurement at the detectors is time translationally invariant and linearly dependent on the measurement time interval $T$.

Including the quantum effects of the homodyne detection in Eq. (9), an individual measurement of an arbitrary field operator $f\left(\left\{\hat{n}_{j}\right\}\right)$ yields the measured value

$$
\left\langle f\left(\left\{\hat{n}_{j}\right\}\right)\right\rangle=\mathcal{N} \sum_{\left\{n_{j}\right\}} f\left(\left\{n_{j}\right\}\right) \operatorname{Tr}\left\{\hat{\rho} \mathcal{P}\left(\left\{n_{j}\right\}\right)\right\},
$$

where the trace is considered over the complete set of states in the density matrix of the initial field $\hat{\rho}=|\psi\rangle_{\text {in in }}\langle\psi|$. With 
Eqs. (9) and (10) representing a general scheme of measurement in the operational approach, we now consider for $f\left(\left\{\hat{n}_{j}\right\}\right)$ the operators of $\left\{\hat{n}_{j}\right\}(j=1,2$ or $3,4,5,6)$,

$$
\hat{\mathcal{E}}_{\theta}(x)=\left(\hat{C}_{\theta}+i \hat{S}_{\theta}\right)^{x}, \quad \hat{\mathcal{E}}_{\phi}(x)=\left(\hat{C}_{\phi}+i \hat{S}_{\phi}\right)^{x} \quad \text { for } x \in \mathrm{R} .
$$

In the construction of $\hat{C}_{\theta}, \hat{S}_{\theta}$ and $\hat{C}_{\phi}, \hat{S}_{\phi}$ pairs in Eqs. (8), the compatibility conditions $\left[\hat{\Sigma}_{i}, \hat{\Sigma}_{j}\right]=0$ of the OQSP ensure that $\left\|\hat{\mathcal{E}}_{\theta}(x)\right\|=1$ and $\left\|\hat{\mathcal{E}}_{\phi}(x)\right\|=1$; hence $\hat{\mathcal{E}}_{\theta}(x)$ and $\hat{\mathcal{E}}_{\phi}(x)$ are unitary operators for all $x \in \mathbb{R}$. According to the procedure outlined in the context of Eqs. (9) and (10), the measurements of these operators yield

$$
\left\langle\hat{\mathcal{E}}_{\theta}(x)\right\rangle=\mathcal{N}_{\theta} \sum_{\left\{n_{j}\right\}}\left[\frac{n_{1}-n_{2}+2 i \sqrt{n_{1} n_{2}}}{n_{1}+n_{2}}\right]^{x}\left\langle\mathcal{P}\left(\left\{n_{j}\right\}\right)\right\rangle
$$

and

$$
\left\langle\hat{\mathcal{E}}_{\phi}(x)\right\rangle=\mathcal{N}_{\phi} \sum_{\left\{n_{j}\right\}}\left[\frac{\left(n_{3}-n_{4}\right)+i\left(n_{5}-n_{6}\right)}{\sqrt{\left(n_{3}-n_{4}\right)^{2}+\left(n_{5}-n_{6}\right)^{2}}}\right]^{x}\left\langle\mathcal{P}\left(\left\{n_{j}\right\}\right)\right\rangle,
$$

where $\left\langle\mathcal{P}\left(\left\{n_{j}\right\}\right)\right\rangle=\operatorname{Tr}\left\{|\psi\rangle_{\text {in in }}\langle\psi| \mathcal{P}\left(\left\{n_{j}\right\}\right)\right\}$. In Eq. (12) $\left\{n_{j}\right\}$ $=\left(n_{1}, n_{2}\right)$ and in Eq. (13) $\left\{n_{j}\right\}=\left(n_{3}, n_{4}, n_{5}, n_{6}\right)$. Clearly, Eq. (12) is well defined if $n_{1}, n_{2}$ are not simultaneously zero and similarly Eq. (13) is well defined if $n_{3}-n_{4}$ and $n_{5}-n_{6}$ are not simultaneously zero in the respective summations above. The idea of the elimination of the configurations $n_{1}$ $=n_{2}=0$, and $n_{3}=n_{4}$ and $n_{5}=n_{6}$ from the statistical weight has been introduced as a crucial element of the operational approach $[2,3,6,9]$ in the implementation of the statistical averages. The effective weight of such configurations becomes non-negligible particularly in the case when the initial field strength is sufficiently weak when the probability of receiving zero photons within the detector's measurement time interval $T$ is finite. For instance, the weight of observing zero photons simultaneously at the detectors $D_{1}, D_{2}$ is given by $\langle\mathcal{P}(0,0)\rangle$. The result of such a null measurement is inconclusive in the calculation of the averages in Eq. (12). Similarly, $n_{3}=n_{4}$ and $n_{5}=n_{6}$ yield additional inconclusive results in the measurement on $\left\langle\hat{\mathcal{E}}_{\phi}(x)\right\rangle$ in Eq. (13). The measured averages are then normalized by excluding the total statistical weight of these inconclusive configurations from the integrated probability. For strong fields, the weight of such ambiguous outcomes is smaller and in the classical field limit there is no contribution from such terms, viz., $\mathcal{N}_{\theta}=\mathcal{N}_{\phi}=1$. In the measurement of the temporal phase the individual fluctuations of these weak components as well as the fluctuations in the relative number of photons can be strong due to the absence of a classical reference source (i.e., a strong local oscillator). Hence the normalization technique introduced by NFM proves to be essential for any operational measurement based on phase and thus also for our approach here. $[2]$

More explicitly, this normalization procedure amounts to

$$
\mathcal{N}_{\theta}^{-1}=1-\langle\mathcal{P}(0,0)\rangle
$$

and

$$
\mathcal{N}_{\phi}^{-1}=1-\sum_{n, m}\left\langle\mathcal{P}\left(n_{3}=n_{4}=n, n_{5}=n_{6}=m\right)\right\rangle
$$

in Eqs. (12) and (13). The observed unitarity conditions of $\hat{\mathcal{E}}_{\theta}(x)$ and $\hat{\mathcal{E}}_{\phi}(x)$ suggest that one can associate a classical random variable $e^{i x \theta}$ and $e^{i x \phi}$ respecting the probability distributions $P(\theta)$ and $P(\phi)$ such that [6]

$$
\left\langle\hat{\mathcal{E}}_{\theta}(x)\right\rangle=\int_{0}^{\pi} d \theta e^{i x \theta} P(\theta), \quad\left\langle\hat{\mathcal{E}}_{\phi}(x)\right\rangle=\int_{-\pi}^{\pi} d \phi e^{i x \phi} P(\phi) .
$$

The probability distributions can then be obtained by the inverse Fourier transformations of Eq. (16) by

$$
P(\theta)=\int_{-\infty}^{\infty} \frac{d x}{2 \pi} e^{-i x \theta}\left\{\left\langle\hat{\mathcal{E}}_{\theta}(x)\right\rangle+\left\langle\hat{\mathcal{E}}_{\theta}(-x)\right\rangle\right\}
$$

$$
P(\phi)=\int_{-\infty}^{\infty} \frac{d x}{2 \pi} e^{-i x \phi}\left\langle\hat{\mathcal{E}}_{\phi}(x)\right\rangle
$$

with $\int_{0}^{\pi} d \theta P(\theta)=\int_{-\pi}^{\pi} d \phi P(\phi)=1$.

Defining two auxiliary functions of $\{n\}_{j}$ by

$$
\theta_{\{n\}}=\tan ^{-1}\left(\frac{2 \sqrt{n_{1} n_{2}}}{n_{1}-n_{2}}\right), \quad \phi_{\{n\}}=\tan ^{-1}\left(\frac{n_{5}-n_{6}}{n_{3}-n_{4}}\right)
$$

where $\{n\}=\left(n_{1}, n_{2}\right)$ and $\{n\}=\left(n_{3}, n_{4}, n_{5}, n_{6}\right)$ for $\theta$ and $\phi$ respectively, and using Eqs. (12) and (13), the moments for a generalized initial state $|\psi\rangle_{\text {in }}$ read

$$
\left\langle\hat{\mathcal{E}}_{\theta}(x)\right\rangle=\mathcal{N}_{\theta} \sum_{n_{1}, n_{2}}{ }^{\prime} e^{i x \theta_{\{n\}}}\left\langle\mathcal{P}\left(\left\{n_{j}\right\}\right)\right\rangle
$$

where

$$
\begin{aligned}
\left\langle\mathcal{P}\left(\left\{n_{j}\right\}\right)\right\rangle= & \mathcal{N}_{\theta} \sum_{n_{1}, n_{2}}{ }^{\prime}\left[\frac{\left(n_{1}-n_{2}\right)+i 2 \sqrt{n_{1} n_{2}}}{n_{1}+n_{2}}\right]^{x} \\
& \times \frac{1}{2^{n_{1}+n_{2}} n_{1} ! n_{2} !} \text { in }\langle\psi|:\left(\hat{a}_{1}^{\dagger} \hat{a}_{1}\right)^{n_{1}}\left(\hat{a}_{2}^{\dagger} \hat{a}_{2}\right)^{n_{2}} \\
& \times \exp \left[-1 / 2\left(\hat{a}_{1}^{\dagger} \hat{a}_{1}+\hat{a}_{2}^{\dagger} \hat{a}_{2}\right)\right]:|\psi\rangle_{\text {in }},
\end{aligned}
$$

and

$$
\left\langle\hat{\mathcal{E}}_{\phi}\left(x ; \delta_{0}\right)\right\rangle=\mathcal{N}_{\phi} \sum_{n_{3}, n_{4}, n_{5}, n_{6}}{ }^{\prime} e^{i x \phi_{\{n\}}\left\langle\mathcal{P}\left(\left\{n_{j}\right\}, \delta_{0}\right)\right\rangle}
$$

where 


$$
\begin{aligned}
\mathcal{P}\left(\left\{n_{j}\right\}\right. & \left., \delta_{0}\right) \\
= & \mathcal{N}_{\phi} \sum_{n_{3}, n_{4}, n_{5}, n_{6}}{ }^{\prime}\left[\frac{\left(n_{3}-n_{4}\right)+i\left(n_{5}-n_{6}\right)}{\sqrt{\left(n_{3}-n_{4}\right)^{2}+\left(n_{5}-n_{6}\right)^{2}}}\right]^{x} \\
& \times \frac{1}{8^{n_{3}+n_{4}+n_{5}+n_{6}} n_{3} ! n_{4} ! n_{5} ! n_{6} !} \\
& \times{ }_{\text {in }}\langle\psi|:\left(\hat{a}_{1}^{\dagger}+\hat{a}_{2}^{\dagger}\right)^{n_{6}}\left(\hat{a}_{1}+\hat{a}_{2}\right)^{n_{3}}\left(-\hat{a}_{1}^{\dagger}+\hat{a}_{2}^{\dagger}\right)^{n_{4}} \\
& \times\left(-\hat{a}_{1}+\hat{a}_{2}\right)^{n_{4}}\left(-i \hat{a}_{1}^{\dagger}+\hat{a}_{2}^{\dagger}\right)^{n_{5}}\left(i \hat{a}_{1}+\hat{a}_{2}\right)^{n_{5}} \\
& \times\left(i \hat{a}_{1}^{\dagger}+\hat{a}_{2}^{\dagger}\right)^{n_{6}}\left(-i \hat{a}_{1}+\hat{a}_{2}\right)^{n_{6}} \\
& \times \exp \left[-1 / 2\left(\hat{a}_{1}^{\dagger} \hat{a}_{1}+\hat{a}_{2}^{\dagger} \hat{a}_{2}\right)\right]:|\psi\rangle_{\mathrm{in}},
\end{aligned}
$$

with $\delta_{0}=\delta_{2}-\delta_{1}$ implicitly described in Eq. (20) as the relative temporal phase between the components of the initial field. The primes on the summations in Eqs. (19) and (20) now indicate that the summations are performed by excluding those configurations for which the outcome is inconclusive.

All moments are now determined once the initial components $\left\langle\hat{n}_{1}\right\rangle,\left\langle\hat{n}_{2}\right\rangle$ and the relative temporal phase $\delta_{0}$ of the inclusive fields $\hat{a}_{1}, \hat{a}_{2}$ are known. In our calculations the initial field parameters are chosen as the ratio of the photon numbers $\eta=\left\langle\hat{n}_{1}\right\rangle /\left\langle\hat{n}_{2}\right\rangle$, the total number of photons $\left\langle\hat{\Sigma}_{0}\right\rangle$ $=\left\langle\hat{n}_{1}\right\rangle+\left\langle\hat{n}_{2}\right\rangle$, and the relative temporal phase $\delta_{0}$.

The credibility of the results obtained from the quantum operational approach crucially depends on the understanding of the influence of the quantum detectors on the final statistics. As pointed out in Refs. [2, 3, 6, 9], another essential element of the operational approach is the construction of an ensemble from a long series of such single operational measurements. The final physical results are then obtained by averaging the outcomes of single measurements over the created ensemble. Based on this prescription, we must now construct a physical ensemble of measured configurations in the calculations of the moments as well as the probability distributions in Eqs. (19) and (20). The response of the quantum detectors to the incoming photons in the creation of the photocurrent is a random process that obeys the Poisson statistics in Eq. (9) [19]. As the photoelectrons are emitted at random times respecting this statistics, the information regarding the initial temporal phase $\delta_{0}$ of the incoming photons is modified and each repeated measurement is equivalent to superposing a random phase shift $\Delta$ on $\delta_{0}$. Hence the process of repeated measurements creates an ensemble of temporal phase configurations $\delta_{0}+\Delta$, with $\Delta$ being uniformly distributed over the available range. Since we consider in our calculations that the measurement time interval $T$ is considerably larger than the coherence time, the available range for $\Delta$ is the entire $2 \pi$ range. Hence the average over the created ensemble corresponds to an averaging over a uniform distribution of $\Delta$. It is clear from Eq. (19) that the moments $\left\langle\hat{\mathcal{E}}_{\theta}(x)\right\rangle$ are independent from $\delta_{0}$; hence they will also be independent of $\Delta$. This implies that a uniform average over $\Delta$ does not influence the measured moments $\left\langle\hat{\mathcal{E}}_{\theta}(x)\right\rangle$ and the probability distribution for $P(\theta)$ is given by

$$
P(\theta)=\int_{-\infty}^{\infty} \frac{d x}{2 \pi} e^{-i x \theta}\left\{\left\langle\hat{\mathcal{E}}_{\theta}(x)\right\rangle+\left\langle\hat{\mathcal{E}}_{\theta}(-x)\right\rangle\right\}, \quad 0 \leqslant \theta \leqslant \pi .
$$

On the other hand, the moments $\left\langle\hat{\mathcal{E}}_{\phi}(x)\right\rangle$ depend on the temporal phase $\delta_{0}$ and before the $\Delta$ average, the $\delta_{0}$ dependence must be replaced by $\delta_{0}+\Delta$. This produces, at each measurement, the conditioned $\phi$ moments $\left\langle\hat{\mathcal{E}}_{\phi}\left(x ; \delta_{0}+\Delta\right)\right\rangle$ and, following Ref. [6], their conditional probability distribution $P\left(\phi, \delta_{0} ; \Delta\right)$ is given by

$$
\begin{gathered}
P\left(\phi, \delta_{0} ; \Delta\right)=\int_{-\infty}^{\infty} \frac{d x}{2 \pi}\left\langle\hat{\mathcal{E}}_{\phi}\left(x ; \delta_{0}+\Delta\right)\right\rangle e^{-i x(\phi-\Delta)}, \\
-\pi \leqslant \phi \leqslant \pi .
\end{gathered}
$$

Therefore, the ensemble-averaged probability distribution is

$$
P\left(\phi, \delta_{0}\right)=\int_{-\pi}^{\pi} \frac{d \Delta}{2 \pi} P\left(\phi ; \delta_{0} ; \Delta\right) .
$$

After a short calculation using Eqs. (19) and (20) in Eqs. (21) $-(23)$, the probability distributions $P(\theta)$ and $P\left(\phi, \delta_{0}\right)$ can be expressed by

$$
P(\theta)=\mathcal{N}_{\theta} \sum_{\left\{n_{j}\right\}} \delta\left(\theta-\theta_{\{n\}}\right)\left\langle\mathcal{P}\left(\left\{n_{j}\right\}\right)\right\rangle
$$

and

$$
P\left(\phi, \delta_{0}\right)=\mathcal{N}_{\phi} \sum_{\left\{n_{j}\right\}}\left\langle\mathcal{P}\left(\left\{n_{j}\right\}, \delta_{0}-\phi_{\{n\}}+\phi\right)\right\rangle,
$$

where the last term in Eq. (25) is obtained by using Eqs. (20) in Eq. (22).

On the other hand, the detectors' influence on the measured statistics can only be understood if the measured moments and probability distributions are compared with those without the detectors' influence. For this purpose and, following Refs. [2,3], we define the theoretically inferred values of the $\theta$ and $\phi$ moments as $\left\langle\hat{\mathcal{E}}_{\theta}^{I}(x)\right\rangle$ and $\left\langle\hat{\mathcal{E}}_{\phi}^{I}(x)\right\rangle$, where

$$
\left\langle\hat{\mathcal{E}}_{\theta}^{I}(x)\right\rangle={ }_{\text {in }}\left\langle\psi\left|:\left[\frac{\hat{n}_{1}-\hat{n}_{2}+i 2 \sqrt{\hat{n}_{1} \hat{n}_{2}}}{\hat{n}_{1}+\hat{n}_{2}}\right]^{x}:\right| \psi\right\rangle_{\text {in }}
$$

and

$$
\left.\left\langle\hat{\mathcal{E}}_{\phi}^{I}(x)\right\rangle={ }_{\text {in }}\left\langle\psi\left|:\left[\frac{\left(\hat{n}_{3}-\hat{n}_{4}\right)+i\left(\hat{n}_{5}-\hat{n}_{6}\right)}{\sqrt{\left(\hat{n}_{3}-\hat{n}_{4}\right)^{2}+\left(\hat{n}_{5}-\hat{n}_{6}\right)^{2}}}\right]^{x}:\right| \psi\right\rangle\right\rangle_{\text {in }},
$$

where : : stands for the normal ordering of the field and vacuum operators inside.

We calculate the probability distributions $P(\theta)$, where 0 $\leqslant \theta \leqslant \pi$, and $P\left(\phi, \delta_{0}\right)$, where $-\pi \leqslant \phi \leqslant \pi$, numerically using Eqs. (24) and (25). Since $\cos \theta$ is single valued in the $\theta$ range considered, we will only need to examine the fluctua- 
tions in the $\hat{C}_{\theta}$ operator. On the other hand, in the $\phi$ range considered both $\hat{C}_{\phi}$ and $\hat{S}_{\phi}$ operators will be necessary. In our calculations, the summations over an infinite range of $\{n\}_{j}$ 's are truncated at $\{n\}_{j}^{\mathrm{max}}=20$ for all $j$ that naturally restrict the accuracy of the results to sufficiently weak initial fields. The measured moments and the probability distributions are then compared with the theoretically inferred ones by using Eqs. (26) and (27).

\section{A. Calculations for a fully polarized quantum coherent field}

Let us now assume that the initial field is in a fully polarized quantum coherent state $|\psi\rangle_{\text {in }}=\left|\alpha_{1}, \alpha_{2}\right\rangle$, with the parameters given by $\alpha_{j}=\left|\alpha_{j}\right| e^{i \delta_{j}}$, where $\left|\alpha_{j}\right|^{2}$ and $\delta_{j}(j=1,2)$ are the average number of photons and the coherent temporal phase of the $j$ th component, respectively. The relative temporal phase is given, as before, by $\delta_{0}=\delta_{2}-\delta_{1}$. From Eqs. (19) and (20), the measured moments in this state are given by

$$
\begin{aligned}
\left\langle\hat{\mathcal{E}}_{\theta}(x)\right\rangle= & \mathcal{N}_{\theta} \sum_{n_{1}, n_{2}}{ }^{\prime}\left[\frac{\left(n_{1}-n_{2}\right)+i 2 \sqrt{n_{1} n_{2}}}{n_{1}+n_{2}}\right]^{x} \\
& \times\left|\alpha_{1}\right|^{2 n_{1}}\left|\alpha_{2}\right|^{2 n_{2}} \\
& \times \exp \left\{-\frac{1}{2}\left(\left|\alpha_{1}\right|^{2}+\left|\alpha_{2}\right|^{2}\right)\right\} / 2^{n_{1}+n_{2}} n_{1} ! n_{2} !
\end{aligned}
$$

and

$$
\begin{aligned}
\left\langle\hat{\mathcal{E}}_{\phi}\left(x ; \delta_{0}\right)\right\rangle= & \mathcal{N}_{\phi} \sum_{\{n\}}{ }^{\prime}\left[\frac{\left(n_{3}-n_{4}\right)+i\left(n_{5}-n_{6}\right)}{\sqrt{\left(n_{3}-n_{4}\right)^{2}+\left(n_{5}-n_{6}\right)^{2}}}\right]^{x} \\
& \times\left|\alpha_{1}+\alpha_{2}\right|^{2 n_{3}}\left|-\alpha_{1}+\alpha_{2}\right|^{2 n_{4}} \mid \\
& -i \alpha_{1}+\left.\alpha_{2}\right|^{2 n_{5}}\left|i \alpha_{1}+\alpha_{2}\right|^{2 n_{6}} \\
& \times \exp \left\{-\frac{1}{2}\left(\left|\alpha_{1}\right|^{2}+\left|\alpha_{2}\right|^{2}\right)\right\} / 8^{n_{3}+n_{4}+n_{5}+n_{6}} \\
& \times n_{3} ! n_{4} ! n_{5} ! n_{6} !,
\end{aligned}
$$

where $\{n\}=\left(n_{3}, n_{4}, n_{5}, n_{6}\right)$. For the specific initial polarized coherent state considered, using Eqs. (14) and (15), the normalizations are given by

$$
\mathcal{N}_{\theta}^{-1}=1-\exp \left\{-\frac{1}{2}\left(\left|\alpha_{1}\right|^{2}+\left|\alpha_{2}\right|^{2}\right)\right\}
$$

and defining $\beta=2\left|\alpha_{1}\right|\left|\alpha_{2}\right| /\left(\left|\alpha_{1}\right|^{2}+\left|\alpha_{2}\right|^{2}\right)$, where $\beta \leqslant 1$,

$$
\begin{aligned}
\mathcal{N}_{\phi}^{-1}= & 1-\sum_{n, m}\left(\frac{\left|\alpha_{1}\right|^{2}+\left|\alpha_{2}\right|^{2}}{8}\right)^{2(n+m)} \\
& \times \frac{\exp \left\{-\frac{1}{2}\left(\left|\alpha_{1}\right|+\left|\alpha_{2}\right|^{2}\right)\right\}}{(n !)^{2}(m !)^{2}} \\
& \times\left[1-\beta^{2} \cos ^{2} \delta_{0}\right]^{n}\left[1-\beta^{2} \sin ^{2} \delta_{0}\right]^{m} .
\end{aligned}
$$

We will first examine the $P(\theta)$ distribution. Using Eq. (24), the calculation of $P(\theta)$ yields

$$
\begin{aligned}
P(\theta)= & \mathcal{N}_{\theta} \sum_{n_{1}, n_{2}}^{\prime} \delta\left(\theta-\theta_{\{n\}}\right) \frac{\left|\alpha_{1}\right|^{2 n_{1}}\left|g a_{2}\right|^{2 n_{2}}}{2^{n_{1}+n_{2}} n_{1} ! n_{2} !} \\
& \times \exp \left\{-\frac{1}{2}\left(\left|\alpha_{1}\right|^{2}+\left|\alpha_{2}\right|^{2}\right)\right\}
\end{aligned}
$$

where $\theta_{\{n\}}$ is defined by the first expression in Eqs. (18). For sufficiently weak fields, i.e., $\left\langle\Sigma_{0}\right\rangle \ll 1$, each detector measures null or a very few number of photons. This implies that in Eq. (32) it is sufficient to restrict the summation over $\left\{n_{1}, n_{2}\right\}$ to a few terms. For instance, let us consider $\left\{n_{1}, n_{2}\right\}=0,1$. Then including only the first-order terms in the average total photon number, Eq. (32) can be approximately expressed in the weak-field limit by $P_{w}(\theta)$ in the form

$$
P_{w}(\theta)=\mathcal{N}_{w}\left\langle\Sigma_{0}\right\rangle\left\{\frac{1}{1+\eta^{-1}} \delta(\theta)+\frac{1}{1+\eta} \delta(\theta-\pi)\right\},
$$

where $\left\langle\Sigma_{0}\right\rangle=\left(\left|\alpha_{1}\right|^{2}+\left|\alpha_{2}\right|^{2}\right) / 2$ is the total average photon number deduced from the measurements at the detectors $D_{1}, D_{2}, \eta=\left|\alpha_{1}\right|^{2} /\left|\alpha_{2}\right|^{2}, \quad$ and $\quad \mathcal{N}_{w}=\left\langle\Sigma_{0}\right\rangle^{-1} \quad$ so that $\int_{0}^{\pi} d \theta P(\theta)=1$. From Eq. (33) we find that

$$
\langle\cos \theta\rangle_{w} \equiv \int_{0}^{\pi} d \theta(\cos \theta) P(\theta)=\frac{\eta-1}{\eta+1}, \quad\left\langle\cos ^{2} \theta\right\rangle_{w}=1 .
$$

Clearly, $\langle\cos \theta\rangle_{w}$ in Eq. (34) is consistent with the theoretically inferred values calculated from Eq. (26) [i.e., $\langle\cos \theta\rangle_{w}$ $\left.=\left\langle\hat{C}_{\theta}^{I}\right\rangle\right]$. In the initial polarized coherent state the theoretically inferred moments are given by

$$
\left\langle\hat{\mathcal{E}}_{\theta}^{I}(x)\right\rangle=\left[\frac{\eta-1+i 2 \eta^{1 / 2}}{\eta+1}\right]^{x}=e^{i x \tan ^{-1} 2 \sqrt{\eta} /(\eta-1)},
$$

which respect a nonfluctuating distribution. Equation (35) is also consistent with the classical calculations using Eq. (4). However, for the second moments we obtain

$$
\left\langle\left(\hat{C}_{\theta}^{I}\right)^{2}\right\rangle=\left(\frac{\eta-1}{\eta+1}\right)^{2} \neq\left\langle\cos ^{2} \theta\right\rangle_{w} .
$$

The $\theta$ distribution in Eq. (32) is plotted in Fig. 3 for $\eta$ $=1.0,0.5$ and $\left\langle\hat{\Sigma}_{0}\right\rangle=0.1,1.0,5.0,10.0$. The first observation in Fig. 3(a) is that at $\eta=1.0$ the probability distribution is symmetrically centered around $\theta=\pi / 2$. In the weak-field limit $P(\theta)$ is peaked at $\theta=0, \pi$. As the field strength is sufficiently increased, the central peak at $\theta=\pi / 2$ gradually develops as all other peaks are suppressed. The average of $\cos \theta$ within the full range $0 \leqslant \theta \leqslant \pi$ is zero, as it would also be expected from the theoretically inferred moments in Eq. (35). For $\eta \neq 1$, the measured $P(\theta)$ is plotted in Fig. 3(b). The $\delta$ functions in Eq. (32) are numerically simulated by sharp Lorentzians, hence they acquire a finite width in Figs. 3(a) and 3(b). On the other hand, using Eq. (21), the inferred probability distribution $P^{I}(\theta)$ can be found as $P^{I}(\theta)=\delta(\theta$ $\left.-\cos ^{-1}(\eta-1) /(\eta+1)\right)$. 

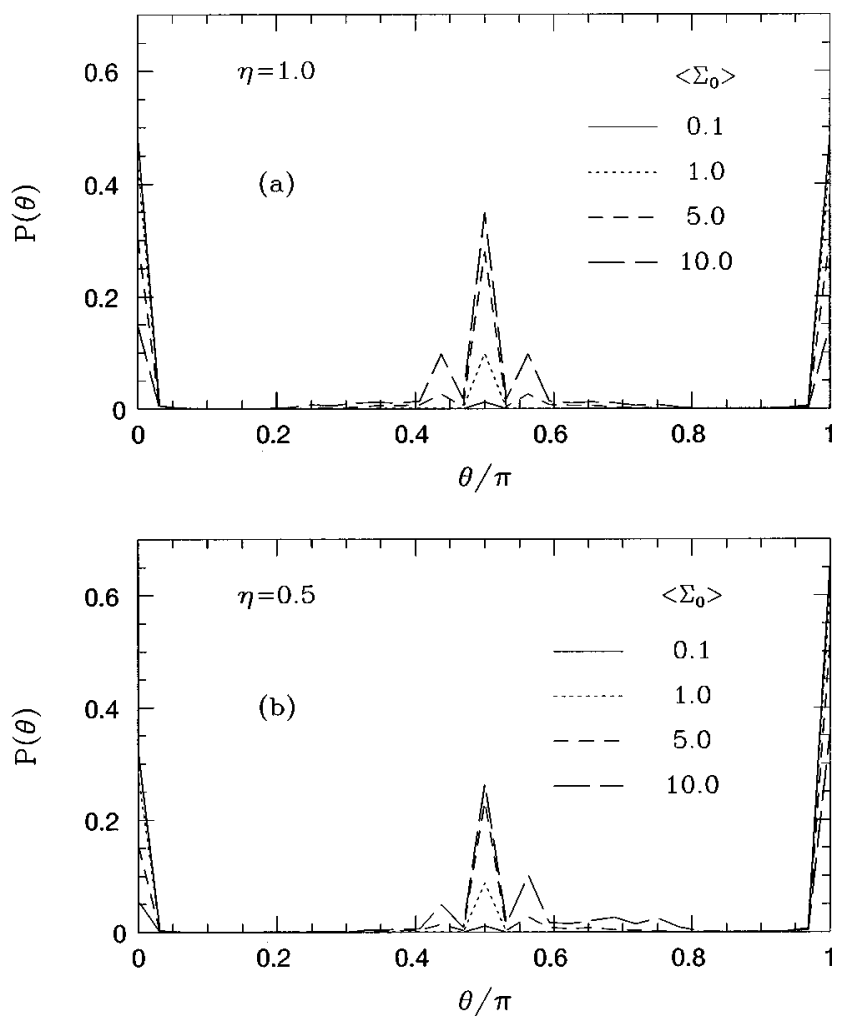

FIG. 3. Measured probability distribution $P(\theta)$ versus $\theta$ for the fully polarized coherent state and (a) $\eta=1.0$ and (b) $\eta=0.5$ for the indicated average total photon numbers.

A similar calculation can also be done for the $P(\phi)$ distribution by making use of Eqs. (29), (22), and (23). After some calculation using the normalization procedure leading to Eq. (23) we find that

$$
\begin{aligned}
P\left(\phi, \delta_{0}\right)= & \mathcal{N}_{\phi_{\left\{n_{j}\right\}}} \sum_{(j=3,4,5,6)} \frac{e^{-\left(\left|\alpha_{1}^{2}\right|+\left|\alpha_{2}\right|^{2} / 2\right.}}{n_{3} ! n_{4} ! n_{5} ! n_{6} !} \\
& \times\left(\frac{\left|\alpha_{1}^{2}\right|+\left|\alpha_{2}\right|^{2}}{8}\right)^{n_{3}+n_{4}+n_{5}+n_{6}} \\
& \times\left[1+\beta \cos \left(\delta_{0}-\phi-\phi_{\{n\}}\right)\right]^{n_{3}} \\
& \times\left[1-\beta \cos \left(\delta_{0}-\phi-\phi_{\{n\}}\right)\right]^{n_{4}} \\
& \times\left[1+\beta \sin \left(\delta_{0}-\phi-\phi_{\{n\}}\right)\right]^{n_{5}} \\
& \times\left[1-\beta \sin \left(\delta_{0}-\phi-\phi_{\{n\}}\right)\right]^{n_{6}},
\end{aligned}
$$

which is, not surprisingly, the same distribution obtained by NFM in Ref. [6] in a slightly different context. The weakfield limit of Eq. (37) has also been studied in Ref. [6], which we refer the reader to for additional details. The numerically calculated equation (37) is plotted in Fig. 4 for $\eta=1.0,0.5$; $\Sigma_{0}=0.1,1.0,5.0,10.0$, and $\delta_{0}=0$. The first observation we make here is that $P\left(20.0, \delta_{0}\right)$ is almost independent from $\eta$ but strongly dependent on the strength of the initial field. As the field strength increases, the fluctuations decrease and the distribution becomes gradually narrower. On the other hand, using Eq. (27), the theoretically inferred moments are calculated as
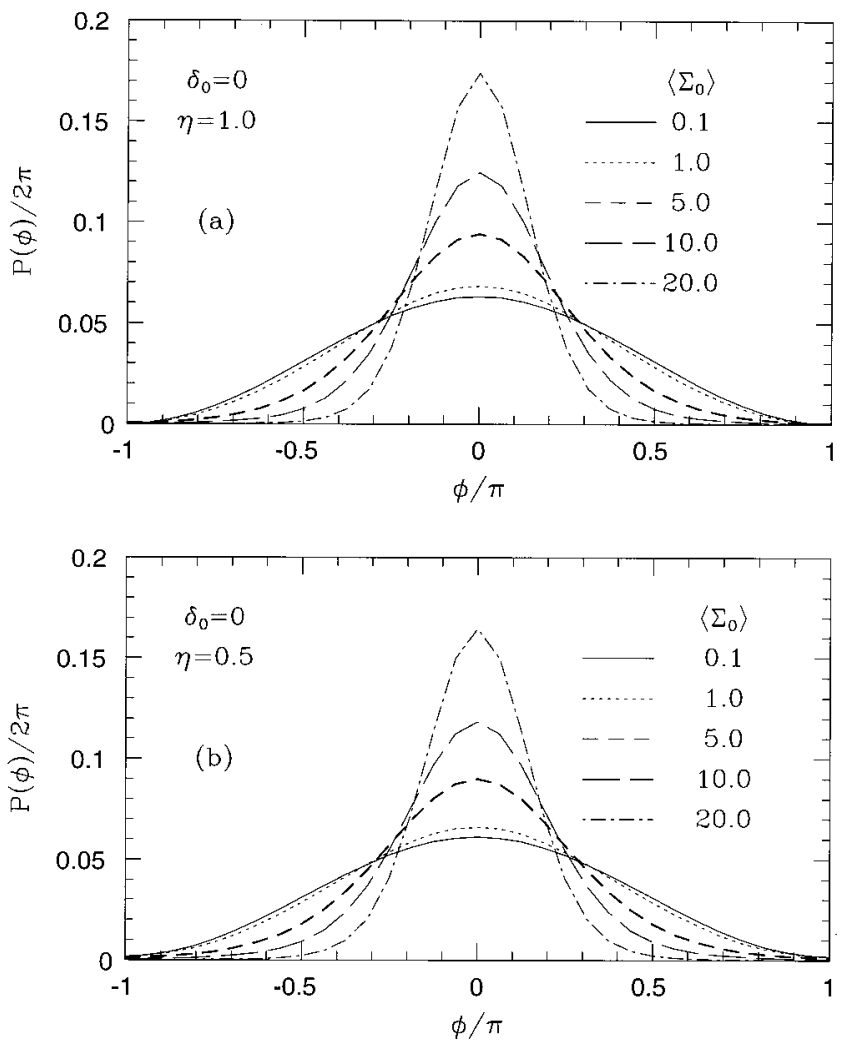

FIG. 4. Measured probability distribution $P(\phi)$ versus $\phi$ for the fully polarized coherent state and (a) $\eta=1.0$ and (b) $\eta=0.5$ for the indicated average total photon numbers.

$$
\left\langle\hat{\mathcal{E}}_{\phi}^{I}(x)\right\rangle=e^{i \delta_{0} x}
$$

Hence the theoretically inferred $\phi$ distribution is also nonfluctuating given by $P\left(\phi, \delta_{0}\right)=\delta\left(\phi-\delta_{0}\right)$. The operational averages for $\phi$ as well as the probability distributions are strongly peaked in the strong-field limit and they have the tendency to approach to the theoretically inferred moments and the $\delta$-function-like probability distributions respectively. On the other hand, the operational approach predicts large deviations of the measurement from the theoretical values in the weak-field limit. In order to understand the influence of the photo-detection particularly in the weak-field limit, we now examine the second-order fluctuations in the measured moments of the $\theta$ and $\phi$ related operators.

\section{Measured fluctuations in polarization}

Once the moments in Eqs. (28) and (29) are defined, the measured moments of the cosine and sine operators of $\theta$ and $\phi$ can be found. The same moments can also be found by the use of the probability distributions in Eqs. (32) and (37). Here the weak-field limit is particularly interesting and can also be examined analytically. We start our analysis of the fluctuations by reminding that, since $0 \leqslant \theta \leqslant \pi$, we will be confined to the measured fluctuations in the $\hat{C}_{\theta}$ operator. In the weak-field limit (keeping only the leading term in the total field strength) 


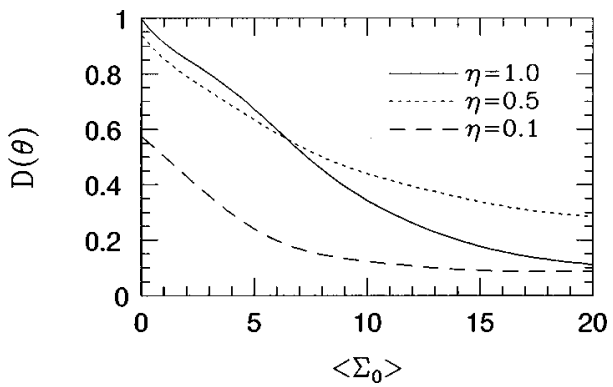

FIG. 5. Second-order fluctuations in the $\theta$ related measurement for the fully polarized coherent state and the indicated values of $\eta$.

$$
\left\langle\hat{C}_{\theta}\right\rangle \simeq \frac{\eta-1}{\eta+1}, \quad\left\langle\hat{C}_{\theta}^{2}\right\rangle \simeq 1,
$$

where we find the dispersion $D(\theta)$ as

$$
D(\theta)=\sqrt{\left\langle\left(\hat{C}_{\theta}-\left\langle\hat{C}_{\theta}\right\rangle\right)^{2}\right\rangle} \simeq \frac{2 \eta^{1 / 2}}{\eta+1} \leqslant 1 .
$$

The dependence of $D(\theta)$ for $\eta=1.0,0.5,0.1$ on the total field strength is shown in Fig. 5. We now shift our attention to the measured fluctuations in the $\phi$ distribution. Since $-\pi \leqslant \phi$ $\leqslant \pi$ we need to consider here both the cosine and the sine moments. Considering first the weak-field limit and keeping only linear terms in the total field strength we have

$$
\begin{aligned}
& \left\langle\hat{C}_{\phi}\right\rangle \simeq \frac{\eta^{1 / 2} \cos \delta_{0}}{\eta+1}, \\
& \left\langle\hat{S}_{\phi}\right\rangle \simeq \frac{\eta^{1 / 2} \sin \delta_{0}}{\eta+1}, \\
& \left\langle\hat{C}_{\phi}^{2}\right\rangle=\left\langle\hat{S}_{\phi}^{2}\right\rangle=\frac{1}{2} .
\end{aligned}
$$

In this case we define the dispersion $D(\phi)$ as

$$
\begin{aligned}
D(\phi) & =\sqrt{\left\langle\left(\hat{C}_{\phi}-\left\langle\hat{C}_{\phi}\right\rangle\right)^{2}\right\rangle+\left\langle\left(\hat{S}_{\phi}-\left\langle\hat{S}_{\phi}\right\rangle\right)^{2}\right\rangle} \\
& =\sqrt{1-\frac{\eta}{(\eta+1)^{2}}} \geqslant \frac{\sqrt{3}}{2} .
\end{aligned}
$$

The dependence of $D(\phi)$ on the total field strength is plotted for $\eta=1.0,0.5,0.1$ in Fig. 6 .

On the other hand, for both the $\theta$ and the $\phi$ related moments the theoretically inferred fluctuations vanish in the co-

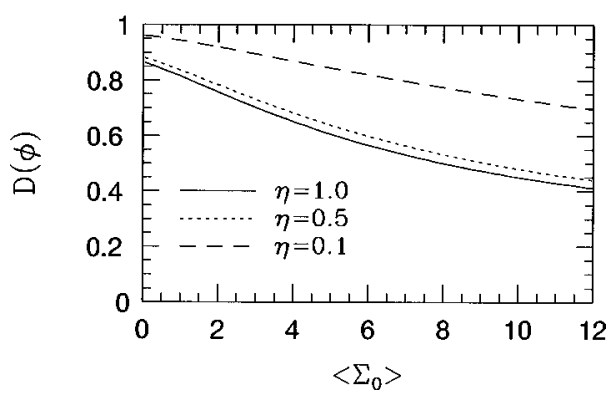

FIG. 6. Second-order fluctuations in the $\phi$ related measurement for the fully polarized coherent state and the indicated values of $\eta$.

herent state [i.e., $D^{I}(\theta)=D^{I}(\phi)=0$ ]. The measured fluctuations differ significantly from the theoretically inferred ones as the strength of the initial field becomes weak. These deviations in Figs. 5 and 6 from the theoretically inferred values arise from the nature of the photodetection of weak fields and the normalization of the probability weight after discarding the inconclusive data. We find that the results for the fully polarized coherent field and the differences between the measured and inferred fluctuations in the weak-field limit closely relate to the results obtained by NFM $[2,6]$.

The $\eta$ dependence of the fluctuations in Figs. 5 and 6 implies that $\eta$ can be used as a parameter in the measurement to search for an optimum orientation of the setup in Fig. 1 by rotating the reference axes 1,2 around the initial field direction. Note that this corresponds to a solid rotation of the entire setup since the relative orientation of each polarizing beam splitter with respect to $\mathrm{PBS}_{1}$ is fixed. By this operation the angle between the polarization axes of the setup and the mean principle axes of the polarization ellipse of the initial field can be changed. Let us suppose that $\alpha_{10}$ and $\alpha_{20}$ are initial coherent state parameters defined with respect to some fixed orientation $1_{0}, 2_{0}$ of $\mathrm{PBS}_{1}$ and given by $\alpha_{j 0}$ $=\left|\alpha_{j 0}\right| e^{i \delta_{j 0} 0}$. If the principle axes 1,2 are rotated by an angle $\gamma$ with respect to $1_{0}, 2_{0}$, the initial coherent field parameters $\alpha_{1}, \alpha_{2}$ are effectively rotated by the same angle with respect to $\alpha_{10}$ and $\alpha_{20}$. In particular, the average number of photons $\left\langle\hat{n}_{1}\right\rangle$ and $\left\langle\hat{n}_{2}\right\rangle$ measured at $D_{1}$ and $D_{2}$ are given by

$$
\begin{aligned}
& \left\langle\hat{n}_{1}\right\rangle=\frac{1}{2}\left|\alpha_{10} \cos \gamma-\alpha_{20} \sin \gamma\right|^{2}, \\
& \left\langle\hat{n}_{2}\right\rangle=\frac{1}{2}\left|\alpha_{20} \cos \gamma+\alpha_{10} \sin \gamma\right|^{2} .
\end{aligned}
$$

Since $\gamma$ is arbitrary, we can use it to tune $\eta=\left\langle\hat{n}_{1}\right\rangle /\left\langle\hat{n}_{2}\right\rangle$ in order to find whether an optimum orientation of the setup exists such that both $\theta$ and $\phi$ related measurements (or whatever other observables are examined) can be improved simultaneously. The measured $\eta$ at the detectors $D_{1,2}$ is then a function of $\gamma$ and is represented in terms of the initially fixed $\eta_{0}=\left|\alpha_{10}\right|{ }^{2} /\left|\alpha_{20}\right|^{2}$ and the relative phase $\left(\delta_{20}-\delta_{10}\right)$ as

$$
\eta(\gamma)=\frac{\left(\sqrt{\eta_{0}}-\tan \gamma\right)^{2}+\sqrt{\eta_{0}} \tan \gamma \sin ^{2}\left(\delta_{20}-\delta_{10}\right) / 2}{\left(\sqrt{\eta_{0}}+\tan \gamma\right)^{2}-\sqrt{\eta_{0}} \tan \gamma \sin ^{2}\left(\delta_{20}-\delta_{10}\right) / 2}
$$



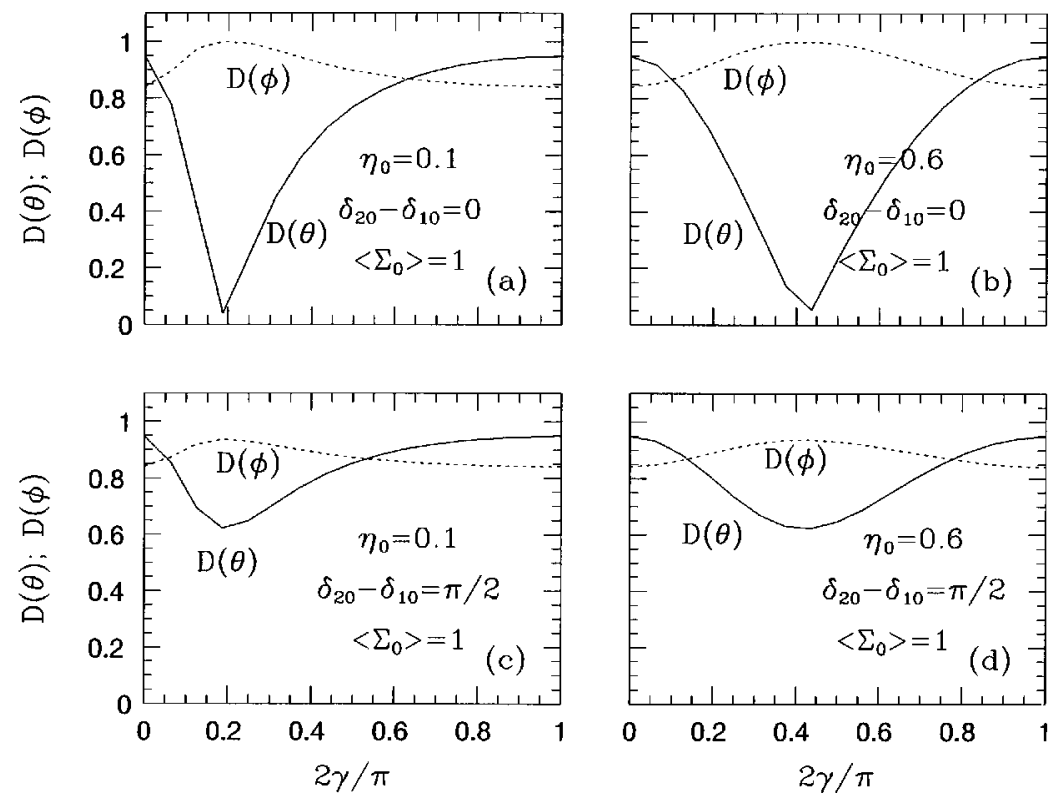

FIG. 7. Second-order fluctuations in the $\theta$ and $\phi$ related measurements for the fully polarized coherent state as a function of the rotation parameter $\gamma$ for the indicated values of $\eta$ and the relative temporal phase in the extreme weak-field limit $\left\langle\Sigma_{0}\right\rangle=1$.
The second-order fluctuations represented by $D(\theta)$ and $D(\phi)$ are plotted in the weak-field limit as a function of $\gamma$ for $0 \leqslant \gamma \leqslant \pi / 2$ and for $\left\langle\Sigma_{0}\right\rangle=1$ in Fig. 7 and for $\left\langle\Sigma_{0}\right\rangle=9$ in Fig. 8. The figures imply that such an optimum orientation to simultaneously minimize the fluctuations $D(\theta)$ and $D(\phi)$ for a fixed value of $\gamma, \delta_{20}-\delta_{10}$, and $\left\langle\Sigma_{0}\right\rangle$ does not exist. Hence, depending on the measured observable, one has to engineer such optimum configurations for each measurement independently.

\section{B. Calculations for a fully polarized Fock state}

Now let us assume that the initial field is given by the fully polarized photon number state $|\psi\rangle_{\text {in }}=|M\rangle_{\phi_{0}}$ as

$$
|M\rangle_{\phi_{0}}=\mathcal{N}_{M} \sum_{m=0}^{M}\left(\begin{array}{c}
M \\
m
\end{array}\right)^{1 / 2} e^{i \phi_{0}(M-m)}|m, M-m\rangle,
$$

where $\phi_{0}$ is a temporal phase between the polarization components, $\mathcal{N}_{M}=2^{-M / 2},\left(\begin{array}{c}M \\ m\end{array}\right)$ is the binomial coefficient, and $|m, M-m\rangle \equiv|m\rangle \otimes|M-m\rangle$ describes the relative number of photons in each component in reference to a particular choice of predefined axes of polarization. It is possible to see that the field operators for the Fock state

$$
\hat{a}_{\phi_{0}}=\frac{1}{\sqrt{2}}\left(\hat{a}_{1}+e^{-i \phi_{0}} \hat{a}_{2}\right), \quad \hat{a}_{\phi_{0}}^{\dagger}=\frac{1}{\sqrt{2}}\left(\hat{a}_{1}^{\dagger}+e^{i \phi_{0}} \hat{a}_{2}^{\dagger}\right)
$$

satisfy

$\hat{a}_{\phi_{0}}|M\rangle_{\phi_{0}}=\sqrt{M}|M-1\rangle_{\phi_{0}}, \quad \hat{a}_{\phi_{0}}^{\dagger}|M\rangle_{\phi_{0}}=\sqrt{M+1}|M+1\rangle_{\phi_{0}}$.

The temporal phase factor $\phi_{0}$ determines the ellipticity of the polarization. If $\phi_{0}=0, \pi$, Eq. (45) provides the basis for linear polarization. For $\phi= \pm \pi / 2$ left and right circularly polarized states are obtained. For arbitrary $\phi_{0}$ left and right elliptically polarized Fock states can be produced. Restrict-
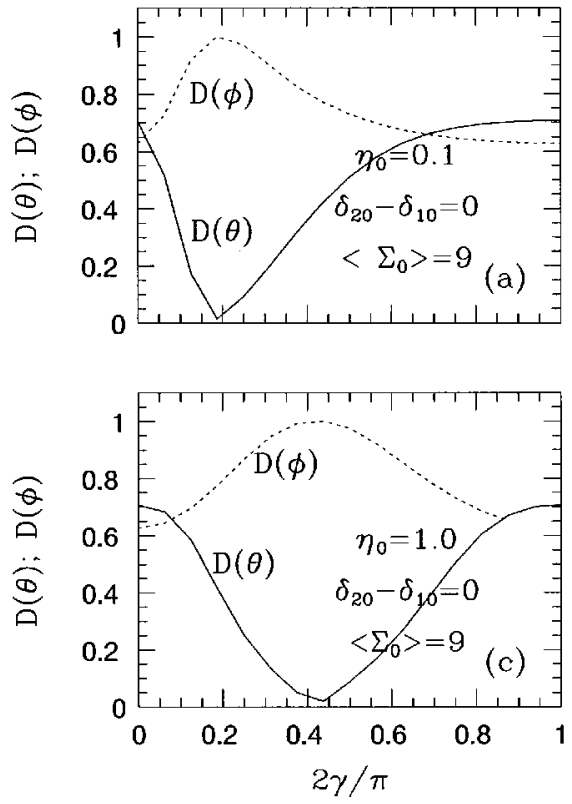

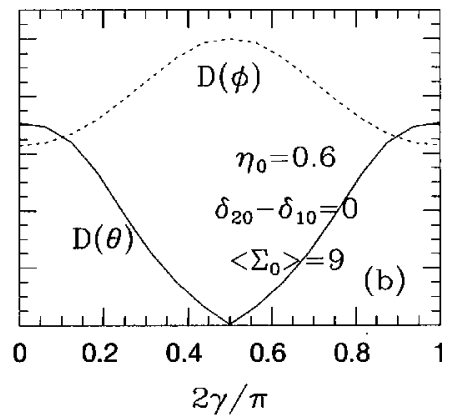

$2 \gamma / \pi$
FIG. 8. Second-order fluctuations in the $\theta$ and $\phi$ related measurements for the fully polarized coherent state as a function of the rotation parameter $\gamma$ for the indicated values of $\eta$ and $\left\langle\Sigma_{0}\right\rangle$ $=9$. 
ing $\phi_{0}$ within the range $0 \leqslant \phi_{0} \leqslant \pi$, the left and right elliptically polarized states are realized respectively by $|M\rangle_{\phi_{0}}$ and $|M\rangle_{\phi_{0}-\pi}$ with the respective field operators $\hat{a}_{\phi_{0}}, \hat{a}_{\phi_{0}}^{\dagger}$ and $\hat{a}_{\phi_{0}-\pi}, \hat{a}_{\phi_{0}-\pi}^{\dagger}$. The second pair of field operators is then found by making the change $\phi_{0} \rightarrow \phi_{0}-\pi$ in Eqs. (46). The field angular momentum operator is given by $\hat{\mathcal{L}}_{z}=\left(\hat{a}_{\phi_{0}}^{\dagger} \hat{a}_{\phi_{0}}\right.$ $\left.-\hat{a}_{\phi_{0}-\pi}^{\dagger} \hat{a}_{\phi_{0}-\pi}\right)$ and $\hat{\mathcal{L}}_{z}$ is diagonal in $|M\rangle_{\phi_{0}}$ with eigenvalue M.

Using Eqs. (46) and (47), Eq. (45) can be written as

$$
|M\rangle_{\phi_{0}}=\frac{1}{\sqrt{M !}}\left(\frac{\hat{a}_{1}^{\dagger}+e^{i \phi_{0}} \hat{a}_{2}^{\dagger}}{\sqrt{2}}\right)^{M}|0,0\rangle=\frac{1}{\sqrt{M !}}\left(\hat{a}_{\phi_{0}}^{\dagger}\right)^{M}|0\rangle,
$$

where $|0\rangle$ is the vacuum state for $\hat{a}_{\phi_{0}}$ as well as for $\hat{a}_{1}$ and $\hat{a}_{2}$ (i.e., $|0\rangle \equiv|0,0\rangle$ ). In what follows, the full range $-\pi \leqslant \phi_{0}$ $\leqslant \pi$ will be considered. In fact, Eq. (45) is an example in a class of fully polarized Fock states corresponding to $\eta$ $=\left\langle\hat{n}_{1}\right\rangle /\left\langle\hat{n}_{2}\right\rangle=1$, where $\left\langle\hat{n}_{1}\right\rangle$ and $\left\langle\hat{n}_{2}\right\rangle$ describe the average number of photons in individual polarization modes. For Eq. (45) we have $\left\langle\hat{n}_{1}\right\rangle=\left\langle\hat{n}_{2}\right\rangle=M / 2$. If a rotation parameter $\gamma$ is introduced [for instance, as in Eq. (43) for the coherent state] in the field space by

$$
\begin{gathered}
\hat{a}_{\phi_{0}, \gamma}=\left(\cos \gamma \hat{a}_{1}+e^{-i \phi_{0}} \sin \gamma \hat{a}_{2}\right), \\
\hat{a}_{\phi_{0}, \gamma}^{\dagger}=\left(\cos \gamma \hat{a}_{1}^{\dagger}+e^{i \phi_{0}} \sin \gamma \hat{a}_{2}^{\dagger}\right)
\end{gathered}
$$

in terms of the new field operators $\hat{a}_{\phi_{0}, \gamma}, \hat{a}_{\phi_{0}, \gamma}^{\dagger}$, the field operators of the initial Fock state in Eq. (45) would be obtained when $\gamma=\pi / 4$ in Eq. (49). This implies that the Fock state $|M\rangle_{\phi_{0}, \gamma}$ created by Eq. (49) is realized effectively by a $\gamma-\pi / 4$ degree rotation of the Fock state in Eq. (45) with $|M\rangle_{\phi_{0}, \gamma}$ being

$$
\begin{aligned}
|M\rangle_{\phi_{0}, \gamma} & =\frac{1}{\sqrt{M !}}\left(\hat{a}_{\phi_{0}, \gamma}^{\dagger}\right)^{M}|0\rangle \\
& =\sum_{m=0}^{M}\left(\begin{array}{c}
M \\
m
\end{array}\right)^{1 / 2}(\cos \gamma)^{m}\left(e^{i \phi_{0}} \sin \gamma\right)^{M-m}|m, M-m\rangle
\end{aligned}
$$

Equation (50) for a fixed $\phi_{0}$ now describes a fully polarized generalized Fock state with an arbitrary ratio of photon numbers $\eta(\gamma)=\cot ^{2} \gamma$ between the polarization components.

In comparison to the coherent initial field, considerably more tedious work is involved in the numerical calculations of both measured moments. In the general fully polarized Fock state given by Eq. (50), Eqs. (12) and (13) become

$$
\begin{aligned}
\left\langle\hat{\mathcal{E}}_{\theta}(x)\right\rangle= & \mathcal{N}_{\theta} \sum_{n_{1}, n_{2}}{ }^{\prime}\left[\frac{\left(n_{1}-n_{2}\right)+i 2 \sqrt{n_{1} n_{2}}}{n_{1}+n_{2}}\right]^{x} \\
& \times \frac{1}{2^{n_{1}+n_{2}} n_{1} ! n_{2} !}\left\{\sum_{r, p} \frac{(-1)^{r+p}}{2^{r+p} r ! p !} \mu_{\left(n_{1}+r\right)}^{\left(n_{2}+p\right)}\right\}
\end{aligned}
$$

and

$$
\begin{aligned}
\left\langle\hat{\mathcal{E}}_{\phi}\left(x, \phi_{0}\right)\right\rangle= & \mathcal{N}_{\phi} \sum_{\{n\}}{ }^{\prime}\left[\frac{\left(n_{3}-n_{4}\right)+i\left(n_{5}-n_{6}\right)}{\sqrt{\left(n_{3}-n_{4}\right)^{2}+\left(n_{5}-n_{6}\right)^{2}}}\right]^{x} \\
& \times \frac{1}{8^{n_{3}+n_{4}+n_{5}+n_{6} n_{3} ! n_{4} ! n_{5} ! n_{6} !}} \\
& \times \sum_{r} \frac{(-1)^{r}}{2^{r} r !}|M|:\left(\hat{a}_{1}^{\dagger} \hat{a}_{1}+\hat{a}_{2}^{\dagger} \hat{a}_{2}\right)^{\tilde{n}+r} \\
& \times\left(1+\frac{\hat{a}_{1}^{\dagger} \hat{a}_{2}+\hat{a}_{2}^{\dagger} \hat{a}_{1}}{\hat{a}_{1}^{\dagger} \hat{a}_{1}+\hat{a}_{2}^{\dagger} \hat{a}_{2}}\right)^{n_{3}} \\
& \times\left(1-\frac{\hat{a}_{1}^{\dagger} \hat{a}_{2}+\hat{a}_{2}^{\dagger} \hat{a}_{1}}{\hat{a}_{1}^{\dagger} \hat{a}_{1}+\hat{a}_{2}^{\dagger} \hat{a}_{2}}\right)^{n_{4}} \\
& \times\left(1-i \frac{\hat{a}_{1}^{\dagger} \hat{a}_{2}-\hat{a}_{2}^{\dagger} \hat{a}_{1}}{\hat{a}_{1}^{\dagger} \hat{a}_{1}+\hat{a}_{2}^{\dagger} \hat{a}_{2}}\right)^{n_{5}} \\
& \left.\times\left(1+i \frac{\hat{a}_{1}^{\dagger} \hat{a}_{2}-\hat{a}_{2}^{\dagger} \hat{a}_{1}}{\hat{a}_{1}^{\dagger} \hat{a}_{1}+\hat{a}_{2}^{\dagger} \hat{a}_{2}}\right)^{n_{6}}: \mid M\right),
\end{aligned}
$$

where in Eq. (51)

$$
\begin{aligned}
\mu_{\left(n_{1}+r\right)}^{\left(n_{2}+p\right)}= & \left\langle M\left|:\left(\hat{a}_{1}^{\dagger} \hat{a}_{1}\right)^{n_{1}+r}\left(\hat{a}_{2}^{\dagger} \hat{a}_{2}\right)^{n_{2}+p}:\right| M\right\rangle \\
= & \sum_{m=0}^{M} \frac{M !}{\left(m-n_{1}-r\right) !\left(M-m-n_{2}-p\right) !} \\
& \times(\cos \gamma)^{2 m}(\sin \gamma)^{2(M-m)},
\end{aligned}
$$

with $n_{1}+n_{2} \leqslant M$ in Eq. (51) and $\tilde{n} \leqslant M$, where $\tilde{n}=n_{3}+n_{4}$ $+n_{5}+n_{6}$ in Eq. (52). In Eq. (52), $\left\langle\hat{\mathcal{E}}_{\phi}\left(x, \phi_{0}\right)\right\rangle$ is understood in the same sense as $\left\langle\hat{\mathcal{E}}_{\phi}\left(x, \delta_{0}\right)\right\rangle$ in Eq. (20). The normalizations are determined as before by satisfying the condition $\left\langle\hat{\mathcal{E}}_{\theta}(0)\right\rangle=\left\langle\hat{\mathcal{E}}_{\phi}\left(0, \phi_{0}\right)\right\rangle=1$.

The simplest analytic results can be obtained for the case $M=1$ with $\gamma$ and $\phi_{0}$ being free parameters. This corresponds for the initial state to

$$
|1\rangle_{\phi_{0}, \gamma}=\cos \gamma|1,0\rangle+e^{i \phi_{0}} \sin \gamma|0,1\rangle,
$$

which is a fully polarized version of the split photon state in Refs. [2, 6]. Using Eqs. (51)-(53), we find for the moments

$$
\left\langle\hat{\mathcal{E}}_{\theta}(x)\right\rangle=\frac{\mathcal{N}_{\theta}}{2}\left[\cos ^{2} \gamma+(-)^{x} \sin ^{2} \gamma\right]
$$

where $\mathcal{N}_{\theta}^{-1}=1 / 2$, and

$$
\begin{aligned}
\left\langle\hat{\mathcal{E}}_{\phi}\left(x, \phi_{0}\right)\right\rangle= & \frac{\mathcal{N}_{\phi}}{8}\left\{\left[1+(-)^{x}+i^{x}+(-i)^{x}\right]\right. \\
& +\left[1-(-)^{x}\right] \sin 2 \gamma \cos \phi_{0} \\
& \left.+\left[i^{x}-(-i)^{x}\right] \sin 2 \gamma \sin \phi_{0}\right\},
\end{aligned}
$$

where $\mathcal{N}_{\phi}^{-1}=1 / 2$. For the probability distributions $P(\theta)$ and $P\left(\phi, \phi_{0}\right)$ we use Eqs. (21)-(25) in the same spirit as we applied to the coherent initial state in Sec. II A. 
A simple calculation yields that

$$
P(\theta)=\cos ^{2} \gamma \delta(\theta)+\sin ^{2} \gamma \delta(\theta-\pi)
$$

and

$$
P\left(\phi, \phi_{0}\right)=\frac{1}{2 \pi}\left\{1+\sin 2 \gamma \cos \left(\phi_{0}-\phi\right)\right\},
$$

where the probability distributions are positive definite and properly normalized, i.e., $\int_{0}^{\pi} d \theta P(\theta)=\int_{-\pi}^{\pi} d \phi P\left(\phi, \phi_{0}\right)$ $=1$. At this point, a crucial limiting case in Eqs. (57) and (58) needs to be mentioned. For $\gamma=\pi / 4$, Eqs. (57) and (58) describe the probability distributions of a fully polarized symmetric Fock state. For $\gamma=0$ and $\pi / 2$ we have singlemode photon Fock states $|1,0\rangle$ and $|0,1\rangle$. The measured probability distributions in these states are $P(\theta)=\delta(\theta)$ and $P\left(\phi, \phi_{0}\right)=1 / 2 \pi$ for $\gamma=0$ and $P(\theta)=\delta(\theta-\pi)$ and
$P\left(\phi, \phi_{0}\right)=1 / 2 \pi$ for $\gamma=\pi / 2$, which correctly describe the statistics of the single-mode Fock state consistently with the theoretical expectations of a uniform distribution for $P\left(\phi, \phi_{0}\right)$. For all other $\gamma$, Eqs. (57) and (58) correctly describe the theoretical distributions for a general $|M\rangle_{\phi_{0}, \gamma}$. This behavior of the probability distributions can also be observed in Eq. (37) in the limits $1 \ll \eta$ and $\eta \ll 1$. The analytic calculations become exponentially harder for $2 \leqslant M$. Nevertheless, explicit forms of the $P(\theta)$ and $P\left(\phi, \phi_{0}\right)$ can be given for a general $M$ as

$$
\begin{aligned}
P(\theta)= & \mathcal{N}_{\theta} \sum_{n_{1}, n_{2}}{ }^{\prime} \delta\left(\theta-\theta_{\{n\}}\right) \frac{1}{2^{n_{1}+n_{2}} n_{1} ! n_{2} !} \\
& \times\left\{\sum_{r, p} \frac{(-1)^{r+p}}{2^{r+p} r ! p !} \mu_{\left(n_{1}+r\right)}^{\left(n_{2}+p\right)}\right\},
\end{aligned}
$$

where Eq. (53) is used, and

$$
\begin{aligned}
& P\left(\phi, \phi_{0}\right)=\mathcal{N}_{\phi} \sum_{\{n\}}{ }^{\prime} \frac{1}{8^{n_{3}+n_{4}+n_{5}+n_{6} n_{3} ! n_{4} ! n_{5} ! n_{6} !}} \sum_{r=0}^{\infty}\left(\frac{-1}{2}\right)^{r} \frac{1}{r !} \sum_{p=0}^{r}\left(\begin{array}{l}
r \\
p
\end{array}\right) \sum_{l_{3}=0}^{n_{3}}\left(\begin{array}{c}
n_{3} \\
l_{3}
\end{array}\right) \sum_{l_{4}=0}^{n_{4}}\left(\begin{array}{c}
n_{4} \\
l_{4}
\end{array}\right) \sum_{l_{5}=0}^{n_{5}}\left(\begin{array}{c}
n_{5} \\
l_{5}
\end{array}\right) \\
& \times \sum_{l_{6}=0}^{n_{6}}\left(\begin{array}{l}
n_{6} \\
l_{6}
\end{array}\right) \sum_{k_{3}=0}^{n_{3}}\left(\begin{array}{l}
n_{3} \\
k_{3}
\end{array}\right) \sum_{k_{4}=0}^{n_{4}}\left(\begin{array}{l}
n_{4} \\
k_{4}
\end{array}\right) \sum_{k_{5}=0}^{n_{5}}\left(\begin{array}{l}
n_{5} \\
k_{5}
\end{array}\right) \sum_{k_{6}=0}^{n_{3}}\left(\begin{array}{l}
n_{3} \\
k_{6}
\end{array}\right)(-1)^{l_{4}+k_{4}}(i)^{l_{6}+k_{5}-l_{5}+k_{6}} \\
& \times \sum_{m=0}^{M} e^{-i\left(\phi_{0}-\phi+\phi_{\{n\}}\right)(\tilde{l}-\tilde{k})} \frac{M !}{(m-\tilde{l}-r+p) !(M-m-p-\tilde{n}+\tilde{l}) !}(\cos \gamma)^{2 m-\tilde{l}+\tilde{k}}(\sin \gamma)^{2(M-m)+\tilde{l}-\tilde{k}},
\end{aligned}
$$

with $\theta_{\{n\}}$ and $\phi_{\{n\}}$ as given by Eqs. (18). The numerical calculations of Eqs. (59) and (60) for linear polarization (e.g., $\left.\phi_{0}=0\right)$, and $\eta=1.0,0.5$ [i.e., corresponding to $\gamma$ $\left.=\pi / 4, \tan ^{-1}(\sqrt{2})\right]$ are presented in Figs. 9 and 10 for various values of $m$. Like in the coherent case, the temporal phase factor $\phi_{0}$ in Eq. (60) only shifts the distribution and does not play any role in the fluctuations. We now shift our attention to the second-order fluctuations in the $\theta$ - and $\phi$-dependent moments.

\section{Measured fluctuations in polarization}

Similar to the coherent state example in Sec. II A, we can examine the $\theta$ and $\phi$ dispersions in the weak-field limit in the range $0 \leqslant \theta \leqslant \pi$ and $-\pi \leqslant \phi \leqslant \pi$ using the same observables as in Sec. II A 1. For $M=1$ we have for $\theta$

$$
\left\langle\hat{C}_{\theta}\right\rangle=\cos 2 \gamma, \quad\left\langle\hat{C}_{\theta}^{2}\right\rangle=1
$$

hence

$$
D(\theta)=\sqrt{\left\langle\left(\hat{C}_{\theta}-\left\langle\hat{C}_{\theta}\right\rangle\right)^{2}\right\rangle}=\sin 2 \gamma=\frac{2 \eta^{1 / 2}}{1+\eta},
$$

and for $\phi$

$$
\left\langle\hat{C}_{\phi}\right\rangle=\frac{1}{2} \sin 2 \gamma \cos \phi_{0},
$$

$$
\begin{gathered}
\left\langle\hat{S}_{\phi}\right\rangle=\frac{1}{2} \sin 2 \gamma \sin \phi_{0}, \\
\left\langle\hat{C}_{\phi}^{2}\right\rangle=\left\langle\hat{S}_{\phi}^{2}\right\rangle=\frac{1}{2},
\end{gathered}
$$

hence

$$
\begin{aligned}
D(\phi) & =\sqrt{\left\langle\left(\hat{C}_{\phi}-\left\langle\hat{C}_{\phi}\right\rangle\right)^{2}\right\rangle+\left\langle\left(\hat{S}_{\phi}-\left\langle\hat{S}_{\phi}\right\rangle\right)^{2}\right\rangle} \\
& =\sqrt{1-\frac{1}{4} \sin ^{2} 2 \gamma}=\sqrt{1-\frac{\eta}{(\eta+1)^{2}}} \geqslant \frac{\sqrt{3}}{2} .
\end{aligned}
$$

It is not an accident that the weak-field limit for the coherent state described in Eq. (42) coincides with the Fock state calculation in Eq. (64) for $M=1$ for all $\eta$. The results of the numerical calculations of $D(\theta)$ and $D(\phi)$ as the initial number of photons is varied are shown for $\eta=1.0,0.1,0.01$ in Fig. 11 for $D(\theta)$, and for $\eta=1.0,0.5,0.1$ in Fig. 12 for $D(\phi)$ corresponding to linear polarization (e.g., $\left.\phi_{0}=0\right)$. Due to the large number of summations in Eq. (60), calculations are considered within the range $1 \leqslant M \leqslant 10$.

The fully polarized Fock state is a typical example where the correlations are present between the relative occupations of the polarization components. As pointed out in Ref. [2], this renders the physical interpretation of the theoretically inferred moments for the $\phi$ related operators impossible. It appears that the operational approach here provides a scheme where the temporal phase distribution can be measured even 

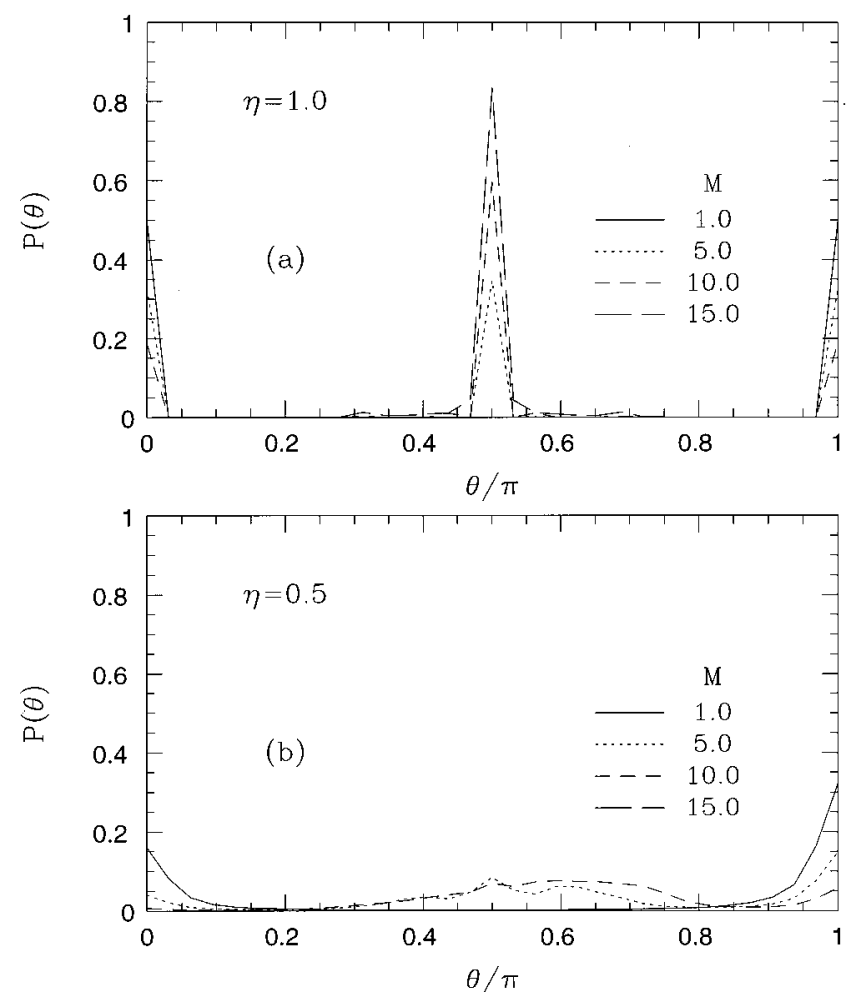

FIG. 9. Measured probability distribution $P(\theta)$ versus $\theta$ for the fully polarized Fock state for (a) $\eta=1$ and (b) $\eta=0.5$ and the indicated average total photon numbers.
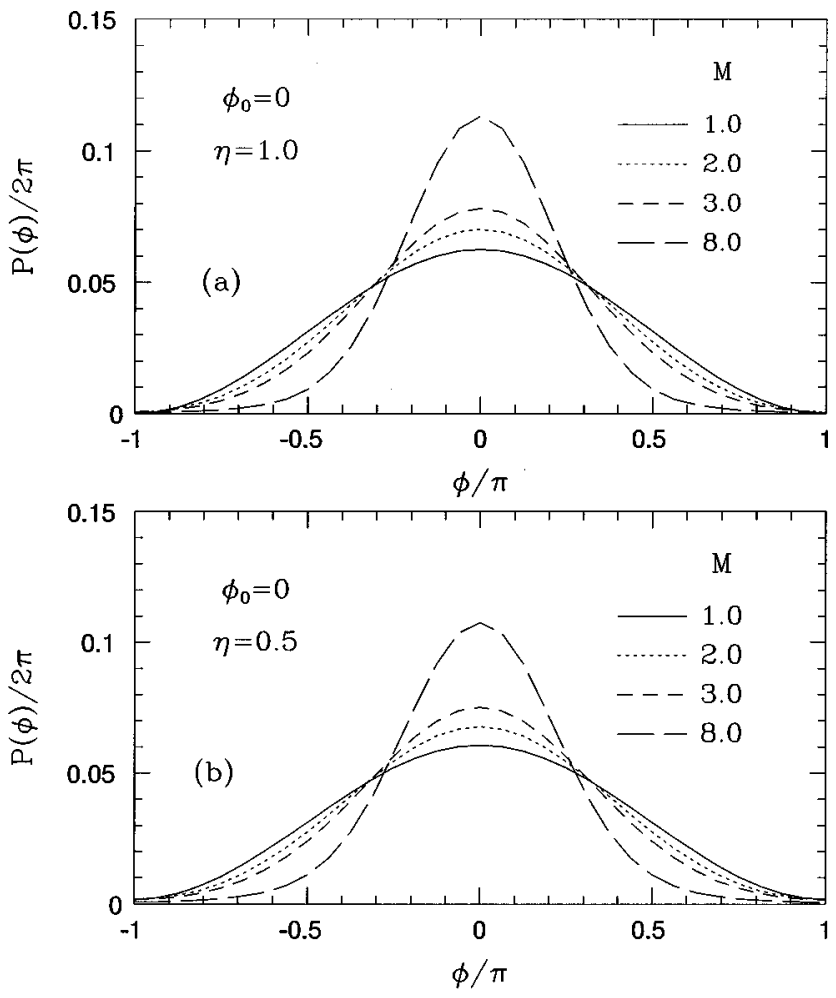

FIG. 10. Measured probability distribution $P(\phi)$ versus $\phi$ for the fully polarized Fock state for (a) $\eta=1$ and (b) $\eta=0.5$ and the indicated average total photon numbers.

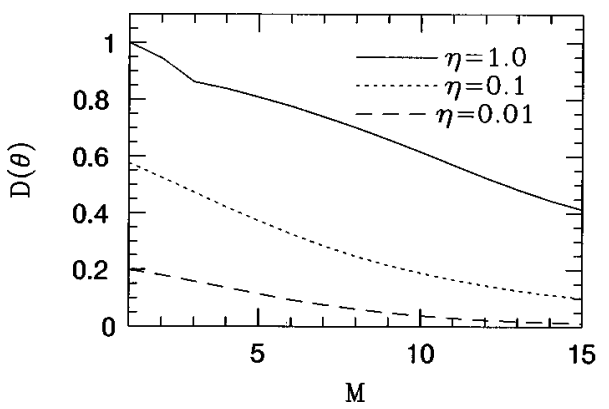

FIG. 11. Second-order fluctuations in the $\theta$ related measurements for the fully polarized Fock state as a function of the average total number of photons and the indicated values of $\eta$.

if such correlations are present. We believe that the results obtained in Figs. 9 and 10 should be checked experimentally with the particular emphasis on the weak-field regimes, which we expect to provide further confirmation of NFM's operational scheme. The split-photon state discussed by NFM in Refs. [2,6] can also be interpreted as the weak-field limit of the polarized Fock state in Eq. (45) corresponding to $M=1$, where strong intensity correlations are present. For this state the inferred moments of the corresponding $\hat{C}_{\phi}$ and $\hat{S}_{\phi}$ are unphysical because of the fact that in Eq. (27) the denominator vanishes. To examine the theoretically inferred moments for a general $M$ we use Eqs. (26) and (27) in the Fock state (45) (we consider $\gamma=\pi / 4$ for simplicity) to calculate

$$
\left\langle\left(\hat{C}_{\theta}^{I}\right)^{x}\right\rangle=\frac{\left\langle M\left|:\left(\hat{n}_{1}-\hat{n}_{2}\right)^{x}:\right| M\right\rangle}{\left\langle M\left|:\left(\hat{n}_{1}+\hat{n}_{2}\right)^{x}:\right| M\right\rangle},
$$

where we find that

$$
\left\langle\hat{C}_{\theta}^{I}\right\rangle=\left\langle\left(\hat{C}_{\theta}^{I}\right)^{2}\right\rangle=0
$$

and

$$
\begin{aligned}
\left\langle\hat{\mathcal{E}}_{\phi}^{I}(x)\right\rangle= & \frac{\left\langle M\left|:\left[\left(\hat{n}_{3}-\hat{n}_{4}\right)+i\left(\hat{n}_{5}-\hat{n}\right)\right]^{x}:\right| M\right\rangle}{\left\langle M\left|:\left[\sqrt{\left(\hat{n}_{3}-\hat{n}_{4}\right)^{2}+\left(\hat{n}_{5}-\hat{n}_{6}\right)^{2}}\right]^{x}:\right| M\right\rangle} \\
= & \frac{\left\langle:\left[\left(\hat{a}_{1}^{\dagger} \hat{a}_{2}+\hat{a}_{2}^{\dagger} \hat{a}_{1}\right)+i\left(\hat{a}_{1}^{\dagger} \hat{a}_{2}-\hat{a}_{2}^{\dagger} \hat{a}_{1}\right)\right]^{x}:\right\rangle}{\left\langle:\left[\sqrt{\hat{a}_{1}^{\dagger} \hat{a}_{2}^{\dagger} \hat{a}_{1} \hat{a}_{2}}\right]^{x}:\right\rangle} .
\end{aligned}
$$

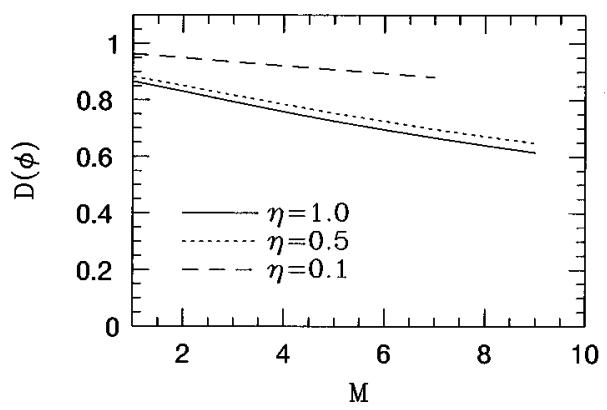

FIG. 12. Second-order fluctuations in the $\phi$ related measurements for the fully polarized Fock state as a function of the total number of photons and the indicated values of $\eta$ (here we considered $\left.\phi_{0}=0\right)$. 
The vacuum fields do not contribute to the normal ordering and we also omitted the state label $M$ in the second step of the expression. In order to calculate Eq. (67) we need $\left\langle:\left[\sqrt{\hat{n}_{1} \hat{n}_{2}}\right]^{x}:\right\rangle$. In the presence of correlations (i.e., $\left\langle\hat{n}_{1} \hat{n}_{2}\right\rangle$ $\left.\neq\left\langle\hat{n}_{1}\right\rangle\left\langle\hat{n}_{2}\right\rangle\right)$ we have

$$
\frac{\left\langle\hat{n}_{1} \hat{n}_{2}\right\rangle}{\left\langle\hat{n}_{1}\right\rangle\left\langle\hat{n}_{2}\right\rangle}=1-\frac{1}{M}<1 .
$$

Hence the correlation effects cannot be ignored if the initial Fock state contains a few photons. Furthermore, Eq. (68) implies that, for $M=1$, the denominator in Eq. (67) diverges at $x=2$. It might therefore be suggested to consider that the comparison with the theoretically inferred moments with the measured operational ones is limited to the strong-field regime $(1 \ll M)$, where also consistency with the classical results are expected to hold. On the other hand, the denominator in Eq. (67) is not well defined for values of $x$ not equal to an even integer. Now let us assume for the moment that we are able to replace the denominator of Eq. (67) by $\left(\left\langle\hat{a}_{1}^{\dagger} \hat{a}_{2}^{\dagger} \hat{a}_{1} \hat{a}_{2}\right\rangle\right)^{x / 2}$. One expects that if this replacement can be done, it can only be valid in the sufficiently strong-field limit where the correlations as well as fluctuations are expected to be negligible. With this replacement, Eq. (67) would yield

$$
\begin{aligned}
& \left\langle\hat{C}_{\phi}^{I}\right\rangle=\frac{\cos \phi_{0}}{\sqrt{1-1 / M}}, \quad\left\langle\hat{S}_{\phi}^{I}\right\rangle=\frac{\sin \phi_{0}}{\sqrt{1-1 / M}}, \\
& \left\langle\left(\hat{C}_{\phi}^{I}\right)^{2}\right\rangle=\cos ^{2} \phi_{0}, \quad\left\langle\left(\hat{S}_{\phi}^{I}\right)^{2}\right\rangle=\sin ^{2} \phi_{0} .
\end{aligned}
$$

The inferred dispersion $D^{I}(\phi)$ calculated from Eqs. (69) is purely imaginary for all $M$, which is an unphysical result. Hence the replacement we made above, in order to make the denominator of Eq. (67) calculable, is unphysical for all $M$; thus it cannot be done. Unlike the coherent state, the comparison with the theoretically inferred moments is made impossible by the presence of strong correlations. Therefore, we are unable to examine the photodetector effects in the weak-field limit in the operational measurement of the fully polarized Fock state using the standard formalism of theoretically inferred moments. The unphysical results we obtained for the inferred moments are not inherent to the quantum scheme. Even in the classical measurement scheme, there is no unique way of extracting the theoretically inferred moments when the relative phase or the relative intensity fluctuations are correlated. We refer the reader to Ref. [2] for a detailed discussion on this topic in the context of operational phase formalism. Nevertheless, we will suggest in the following subsection that for the Fock state in Eq. (50), or specifically for Eq. (45), it is possible to find another measure to examine the photodetector effects in the weak-field limit by making use of the properties of the uncertainty relations.

\section{Fully polarized Fock state and connections to the $\operatorname{su}(2)$ interferometry}

For symmetric distribution of photon numbers in the components of the polarization, the fully polarized Fock state in Eq. (50) becomes Eq. (45), which is a generalized su(2) coherent state [20]

$$
\begin{aligned}
\left|j_{0} \xi\right\rangle & =e^{\left(\dot{\xi} \hat{J}_{+}-\xi^{*} \hat{J}_{-}\right)}\left|j_{0},-j_{0}\right\rangle \\
& =\frac{1}{\left(1+|\xi|^{2}\right)^{j_{0}}} \sum_{n=-j_{0}}^{j_{0}}\left(\begin{array}{c}
2 j_{0} \\
j_{0}+n
\end{array}\right)^{1 / 2} \xi^{j_{0}+n}\left|j_{0} n\right\rangle,
\end{aligned}
$$

which becomes clear if one makes a correspondence between Eqs. (70) and (45) as

$$
j_{0} \rightarrow M / 2, \quad j_{0}-n \rightarrow m, \quad j_{0}+n \rightarrow M-m, \quad \xi \rightarrow e^{i \phi_{0}}
$$

or

$$
j_{0}+n \rightarrow m, \quad j_{0}-n \rightarrow M-m, \quad \xi \rightarrow e^{-i \phi_{0}},
$$

where in Eq. (70) $\left|j_{0} n\right\rangle=\left|j_{0}-n, j_{0}+n\right\rangle$. Here $\hat{J}_{ \pm}=\hat{J}_{1} \pm i \hat{J}_{2}$ are the standard raising and lowering operators of the $\mathrm{su}(2)$ angular momentum algebra defined by the generators $\hat{J}_{i}(i$ $=0, \ldots, 3)$,

$$
\begin{aligned}
& \hat{J}_{0}=\left(\hat{a}_{1}^{\dagger} \hat{a}_{1}+\hat{a}_{2}^{\dagger} \hat{a}_{2}\right) / 2, \\
& \hat{J}_{1}=\left(\hat{a}_{1}^{\dagger} \hat{a}_{1}-\hat{a}_{2}^{\dagger} \hat{a}_{2}\right) / 2, \\
& \hat{J}_{2}=\left(\hat{a}_{1}^{\dagger} \hat{a}_{2}+\hat{a}_{2}^{\dagger} \hat{a}_{1}\right) / 2, \\
& \hat{J}_{3}=\left(\hat{a}_{1}^{\dagger} \hat{a}_{2}-\hat{a}_{2}^{\dagger} \hat{a}_{1}\right) / 2 i,
\end{aligned}
$$

where, considering that $\left(\hat{a}_{1}^{\dagger}, \hat{a}_{1}\right),\left(\hat{a}_{2}^{\dagger}, \hat{a}_{2}\right)$ represent two independent boson pairs, we have the standard su(2) algebra $\left[\hat{J}_{i}, \hat{J}_{j}\right]=i \epsilon_{i j k} \hat{J}_{k}(i, j, k=1,2,3)$. Here the central invariant of the algebra is $\hat{J}^{2}=\hat{J}_{0}\left(\hat{J}_{0}+1\right)=j_{0}\left(j_{0}+1\right)$, where $j_{0}=M / 2$ with $M$ describing the total number of particles in the Fock state (45). The uncertainty relations for the $\hat{J}_{i}$ 's are given by

$$
\left(\Delta \hat{J}_{i}\right)\left(\Delta \hat{J}_{j}\right) \geqslant \frac{\left|\epsilon_{i j k}\right|}{2}\left\langle J_{k}\right\rangle, \quad i \neq j \neq k=1,2,3 .
$$

Hence, the fully polarized Fock state is nothing but the generalized coherent state of the free field su(2) angular momentum algebra. Under certain conditions Eq. (70) also coincides with the su(2) minimum uncertainty states [21-23] minimizing Eq. (73) which has been explored recently in the current literature in the context of su(2) interferometry [22-24].

The idea of su(2) interferometry is to create interference between two arbitrary input fields by using passive and active lossless optical devices to measure the relative temporal phase between the fields. For this purpose the measured operators of the su(2) interferometry are defined as in Eqs. (72) or they are related to Eqs. (72) by certain unitary transformations induced by the passive and active optical devices. These transformations of Eqs. (72) (or the inverse transformations on the initial fields) can be engineered in such a way that the relative phase shift between the input fields can be measured by pure intensity measurements on the fields at the output ports of the interferometer [22]. The principles of the quantum interferometry are thus based on a generalized operational scheme that is, in principle, very similar to the idea of the operational phase measurement presented in Refs. $[2,3,6,9]$ as well as the present work. 

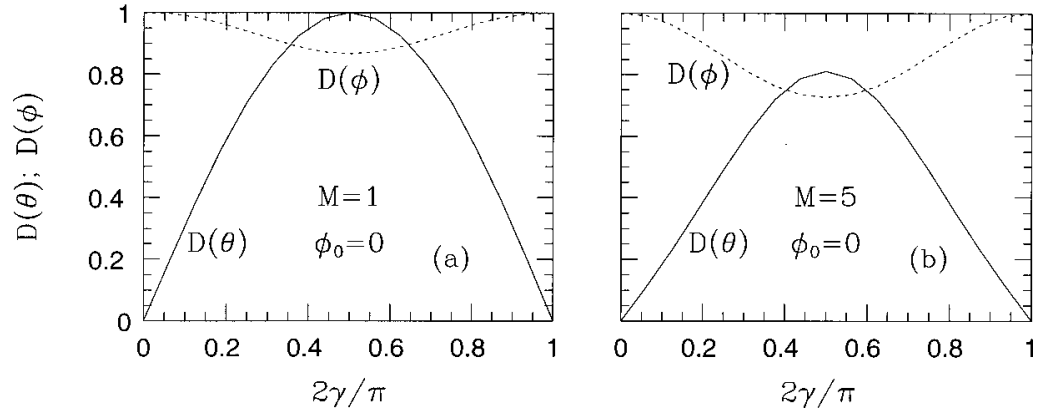

FIG. 13. Second-order fluctuations in the $\theta$ and $\phi$ related measurements for the fully polarized Fock state as a function of the rotation parameter $\gamma$ for the indicated values of $M$ and $\phi_{0}$.
Now let us construct the uncertainty product for the general fully polarized Fock state $|M\rangle_{\phi_{0}, \gamma}$ and particularly focus our attention on the specific limit $|M\rangle_{\phi_{0}}$ at $\gamma=\pi / 4$. The measured interferometric operators correspond, in the standard su(2) interferometry, to the expected values of the operators in Eqs. (72) or some linear superpositions of them in the initial state. For $|M\rangle_{\phi_{0}, \gamma}$ being the initial state, we have

$$
\begin{gathered}
\left\langle\hat{J}_{0}\right\rangle=\frac{M}{2}, \\
\left\langle\hat{J}_{1}\right\rangle=\frac{M}{2} \cos 2 \gamma, \\
\left\langle\hat{J}_{2}\right\rangle=\frac{M}{2} \sin 2 \gamma \cos \phi_{0}, \\
\left\langle\hat{J}_{3}\right\rangle=\frac{M}{2} \sin 2 \gamma \sin \phi_{0}
\end{gathered}
$$

and

$$
\begin{gathered}
\left(\Delta \hat{J}_{0}\right)^{2}=0, \\
\left(\Delta \hat{J}_{1}\right)^{2}=\frac{M}{4} \sin ^{2} 2 \gamma, \\
\left(\Delta \hat{J}_{2}\right)^{2}=\frac{M}{4}\left(1-\cos ^{2} \phi_{0} \sin ^{2} 2 \gamma\right), \\
\left(\Delta \hat{J}_{3}\right)^{2}=\frac{M}{4}\left(1-\sin ^{2} \phi_{0} \sin ^{2} 2 \gamma\right) .
\end{gathered}
$$

Using $\gamma=\pi / 4$ in Eqs. (75) and (74) we observe that $|M\rangle_{\phi_{0}}$ is an important state in the algebra defined by the operators in Eq. (72). It is an su(2) coherent state [see Eq. (70)] as well as a minimum uncertainty (intelligent) state minimizing $\mathrm{Eq}$. (73) for $i \neq j=1,3 ; k=2$ and $i \neq j=1,2 ; k=3$. This can be explicitly seen by using Eqs. (74) and (75) in Eq. (73). Furthermore, when $\gamma=\pi / 4$, this result is independent from $\phi_{0}$; hence a temporal shift in $\phi_{0}$ does not change any of these properties. This implies that if $\mathrm{su}(2)$ interferometric techniques [22] are employed for $|M\rangle_{\phi_{0}}$, the standard precision can be achieved in the measurement of the temporal phase $[23,24]$. The precision in the phase measurement can be found from Eqs. (74) and (75) for the general case with $|M\rangle_{\phi_{0}, \gamma}$ as

$$
\delta \phi_{0}(\gamma)=\frac{\left(\Delta \hat{J}_{3}\right)}{\left|\partial\left\langle\hat{J}_{3}\right\rangle / \partial \phi_{0}\right|}=\frac{1}{\sqrt{M}} \frac{\sqrt{1-\sin ^{2} 2 \gamma \sin ^{2} \phi_{0}}}{\sin 2 \gamma \cos \phi_{0}}
$$

where $\delta(\gamma) \geqslant \delta \phi_{0}(\pi / 4)=1 / \sqrt{M}$, which is the well-known minimum standard noise limit. Hence, theoretically, the maximum precision in the phase measurement can be achieved only at $\gamma(\pi / 4)=1$ corresponding to $\eta=1$. The basic idea being the extraction of the phase statistics from pure photon counting, the $\mathrm{su}(2)$ interferometry is in close analogy to the operational measurement scheme. The operators $\hat{J}_{i}(i$ $=0,1,2,3)$ are the interferometric analogs of the operational ones $\hat{\Sigma}_{i}(i=0,1,2,3)$ in Eq. (7), but there are also significant differences between them. Although the $\hat{J}_{i}$ 's are the generators of the $\mathrm{su}(2)$ algebra, the $\hat{\Sigma}_{i}$ 's all commute with each other and no useful uncertainty product similar to Eq. (73) can be written for them. Now a legitimate question arises as to how much the properties of the quantum state $|M\rangle_{\phi_{0}, \gamma}$, as far as the $\hat{J}_{i}$ 's are concerned, are preserved in the operational measurement scheme using the $\hat{\Sigma}_{i}$ operators. The main difference arising from the presence of the vacuum states in the $\hat{\Sigma}_{i}$ 's as well as the operational scheme itself, it is nevertheless expected that for sufficiently strong fields the quantum operational measurement using the $\hat{\Sigma}_{i}$ operators should be consistent with Eq. (73). The deviations in the quantum operational measurement scheme from Eq. (73) are expected when the initial field is sufficiently weak. Hence, by examining the uncertainty properties of $|M\rangle_{\phi_{0}, \gamma}$, particularly near $\gamma=\pi / 4$, a perfect ground to understand the influence of the operational scheme in the final measurement can be provided.

We start the analysis of the uncertainty relations for $|M\rangle_{\phi_{0}, \gamma}$ by examining the $\gamma$ dependence of the measured $D(\theta)$ and $D(\phi)$. The results are represented in Fig. 13 for $M=1,5$ and linear polarization in the range $0 \leqslant \gamma \leqslant \pi / 2$. The figure indicates that, similarly to the results obtained for the fully polarized coherent state measurements, it is not possible to simultaneously minimize the fluctuations in the measurements of the $\theta$ and $\phi$ related moments. The values of $D(\theta)$ and $D(\phi)$ in Fig. 13 corresponding to $\gamma=\pi / 4$ (i.e., $\eta=1$ ), $\gamma \simeq 0.3 \pi$ (i.e., $\eta=0.5$ ), $\gamma \simeq 0.4 \pi$ (i.e., $\eta=0.1$ ), and $\gamma \simeq 0.47 \pi$ (i.e., $\eta=0.01$ ) can also be seen in Figs. 11 and 12 . Here $\gamma=\pi / 4$ has a special importance since this point corresponds to where $|M\rangle_{\phi_{0}, \gamma}$ becomes a coherent as well as a minimum uncertainty state of the $\hat{J}_{i}$ 's. As $\gamma$ is shifted away 


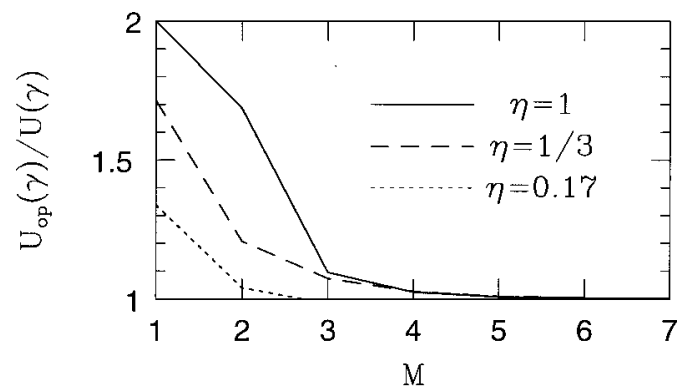

FIG. 14. Comparison between the theoretical uncertainty product and the measured one in the fully polarized Fock state for the indicated values of $\eta$.

from $\pi / 4$, particularly towards $\gamma=0, \pi / 2$, one particular polarization mode starts dominating where the initial state gradually starts looking like a single-mode Fock state. The single-mode Fock limit is realized at $\gamma=0, \pi / 2$ for $\theta$ related measurements [i.e., $D_{\theta}=0$ and $P(\theta)$ comprises a single $\delta$-function peak] and maximally random fluctuations are observed in the $\phi$ related measurements [i.e., $D_{\phi}=1$ and $P(\phi)=1 / 2 \pi]$. Because of the fact that the interferometric operators do not commute with each other, it is not possible to find the interferometric analogs of the trigonometric operators $\hat{C}_{\theta}, \hat{S}_{\theta}$ and $\hat{C}_{\phi}, \hat{S}_{\phi}$ defined in Eqs. (8). This implies that the interferometric analogs of $D_{\theta}$ and $D_{\phi}$ cannot be found by direct analogy and a comparison between the theory and the measurement is not possible for them. At this level, the only comparison with the theory can be made by examining the minimum uncertainty product for the $\hat{J}_{i}$ 's and the $\hat{\Sigma}_{i}$ 's.

Keeping $\gamma$ as the parameter, we now express Eq. (73) in $|M\rangle_{\phi_{0}, \gamma}$ in the form

$$
\mathcal{U}(\gamma)=\frac{\left(\Delta \hat{J}_{2}\right)^{2}+\left(\Delta \hat{J}_{3}\right)^{2}}{\left\langle\hat{J}_{2}\right\rangle^{2}+\left\langle\hat{J}_{3}\right\rangle^{2}}\left(\Delta \hat{J}_{1}\right)^{2}
$$

and find from Eqs. (74) and (75) that

$$
\mathcal{U}(\gamma)=\frac{1}{2}\left(1-\frac{1}{2} \sin ^{2} 2 \gamma\right)
$$

with the minimum uncertainty corresponding to $\mathcal{U}(\pi / 4)$ $=\frac{1}{4}$. The operational analog of Eq. (77) in terms of the $\hat{\Sigma}_{i}$ 's can be found by direct inspection of Eqs. (5)-(7) and (72) as

$$
\mathcal{U}_{\mathrm{op}}(\gamma)=\frac{\left(\Delta \hat{\Sigma}_{2}\right)^{2}+\left(\Delta \hat{\Sigma}_{3}\right)^{2}}{\left\langle\hat{\Sigma}_{2}\right\rangle^{2}+\left\langle\hat{\Sigma}_{3}\right\rangle^{2}}\left(\Delta \hat{\Sigma}_{1}\right)^{2},
$$

with all fluctuations in Eq. (79) calculated within the operational scheme outlined in Sec. II. In comparing Eq. (79) with Eq. (77) the differences arising from the different normalizing factors of the transmission and the reflection coefficients in the $\hat{d}_{i}$ 's in Eq. (5) should also be accounted for. The result of the numerical calculations for $\mathcal{U}_{\mathrm{op}}(\gamma) / \mathcal{U}(\gamma)$ is presented in Fig. 14 as a function of $M$ for various $\eta$ values where $\eta(\gamma)=\cot ^{2} \gamma$. We also observed that Eq. (79) has no $\phi_{0}$ dependence for all $M$ and $\gamma$ (not shown in Fig. 14), which is consistent with the theoretical calculation in Eq. (78).
Figure 14 indicates that the photodetection in the operational scheme unavoidably creates an additional noise in the measurement such that the theoretical value of the uncertainty is not reached until the initial state has a sufficiently large (i.e., $5 \leq M$ ) number of photons. Here, as $\gamma$ varies, there is a compromise between the value of the measured uncertainty product for large $M$ and the detector noise for small $M$. For instance, at $\gamma=\pi / 4$ (i.e., $\eta=1$ ), the measured uncertainty product approaches the theoretical minimum uncertainty for large $M$, although the detector noise is as large as $100 \%$ at the small- $M$ limit. On the other hand, as $\gamma$ deviates from $\pi / 4$, the measured uncertainty product is no longer at the minimum for large $M$, but the detector noise is smaller for small $M$. Hence it appears that there is no global optimum value for $\gamma$. We thus conclude that $\gamma$ can be optimally fixed only depending on the individual observables chosen in the measurement (i.e., a result that we have also reached in the fully polarized coherent state example in Sec. II A).

In the theoretical interferometric calculations it is a common practice to neglect the influence of the photodetection. This is certainly a valid assumption if the initial field is sufficiently strong. On the other hand, we expect the additional noise in the uncertainty product to be a manifestation of any scheme based on photon counting in the weak-field limit arising from the quantum nature of the photodetection. Hence it is also natural to expect these effects to be observable in the su(2) interferometric measurements. This result indeed needs experimental verification, particularly considering the advantage that certain schemes have been proposed for the generation of such quantum states as Eq. (45) experimentally using active nonlinear processes [25].

\section{DISCUSSION}

In this work we focused our attention on the operational measurement scheme as applied to certain fully polarized quantum states particularly in the weak-field regime. We have shown that, similarly to NFM's operational phase measurement scheme, it is possible to base the measurement of the state of polarization on pure photocount measurements, hence providing another example for an operational approach. In particular, the measurement of the fluctuations of the temporal phase between the polarized field components is, not surprisingly, identical to the original work by NFM. The statistical behavior of the Stokes parameters is investigated in terms of the trigonometric operators in Eqs. (8) and the operational counterparts of the quantum Stokes parameters of the polarized field are introduced in Eqs. (7). The application of the operational polarization measurement scheme is made to fully polarized quantum coherent as well as Fock states. With the purpose of extracting the detectors' influence on the measurement, the statistics of the measured fluctuations are examined and compared with the theoretical calculations. Our results confirm those of NFM's operational phase measurement scheme to conclude that the photoncounting process introduces additional noise in the final statistics particularly in the weak-field regimes. For sufficiently strong fields, the operational measurement scheme is consistent with those theoretical predictions in which the photodetection effects are not included.

The connection between the operational approach to the 
measurement of polarization and the $\mathrm{su}(2)$ interferometry is examined and the uncertainty principle is used as a means of analyzing the photodetection effects in the measurement where applications are made on the fully polarized Fock state. The operational approach to the measurement of phase has been investigated by D'Ariano and Paris [26] in the context of quantum estimation theory [27], which provides a unified formulation of the measurement process and the initial system under investigation. The quantum probability distribution of the $N$-port homodyne detection in Eq. (9) is a specific example of the probability-operator-valued measure (POM) in the quantum estimation theory. An ideal quantum measurement is realized when the POM is based on an orthogonal and complete set of states comprising the eigenspace of the measured observable. Hence, depending on the nature of the measured observables of the initial state, finding an optimum detection scheme is the primary goal of a unified formulation of the measurement and the initial system. For the measured observable being phase related quantities, such an approach has not been idealized yet because an orthogonal POM cannot be physically realized for the phase observable. With this in mind, one resorts to optimizing the phase measurement by a proper choice of the initial states as well as the parameters of the measuring system. At this point a connection is present between the primary goal of the quantum estimation theory and the attempts to surpass the standard noise limit by using interferometric transformations on the measuring system (or inverse transformations on the initial state). The optimal choice of these transformations using active as well as passive optical devices naturally depends on the initial state. Furthermore, it is also desirable (and under certain conditions strictly required) to have the transformed state conserve the basic features of the original state, i.e., full polarization, coherent and minimum uncertainty states for Eq. (45), the statistics of the fluctuations, etc. It is natural that for the initial state being fully polarized, the full polarization itself is a strict condition that should be conserved by the transformations. On the other hand, since $|M\rangle_{\phi_{0}}$ is a minimum uncertainty state, the quantum statistics of the temporal phase $\phi$ and the fluctuations in corresponding $\phi$-dependent operators are coupled with those describing the fluctuations in the relative photon number $\hat{n}_{1}-\hat{n}_{2}$. Hence the minimum uncertainty condition of $|M\rangle_{\phi_{0}}$ will most certainly be at stake after such transformations and this will change the quantum nature of the state [for instance, a rotation in the field space by $\gamma$ does not change the full polarization property but changes the minimum uncertainty relations, as it can be seen from Eqs. (73) and (78)]. For those states that are not the minimum uncertainty ones, this observation is still valid to a lesser extent. We nevertheless conclude that attempts to surpass the standard noise limit for the fully polarized quantum states have to comply with a number of restrictions, which certainly renders it a rather interesting problem.
[1] S. M. Barnett and D. T. Pegg, J. Phys. A 19, 3849 (1986).

[2] J. W. Noh, A. Fougères, and L. Mandel, Phys. Rev. A 45, 424 (1992).

[3] J. W. Noh, A. Fougères, and L. Mandel, Phys. Rev. A 46, 2840 (1992).

[4] W. Vogel and W. Schleich, Phys. Rev. A 44, 7642 (1991).

[5] R. Lynch, Phys. Rev. A 47, 1576 (1993).

[6] J. W. Noh, A. Fougères, and L. Mandel, Phys. Rev. Lett. 71, 2579 (1993).

[7] Z. Hradil, Phys. Rev. A 47, 4532 (1993).

[8] D. T. Pegg and S. M. Barnett, Phys. Rev. A 47, 4537 (1993).

[9] J. W. Noh, A. Fougères, and L. Mandel, Phys. Rev. A 47, 4535 (1993).

[10] Here the author believes that one should make a clear distinction between two different major perspectives dominating the field of quantum phase research. The first perspective is to consider the quantum phase as a property of quantum mechanics within a generalized quantum-phase-space formalism. This was the original stand taken by Dirac in his search for a correspondence between the classical canonical variables in the action-angle phase space and their quantum counterparts [see P. A. M. Dirac, Proc. R. Soc. London, Ser. A 114, 243 (1927); and also P. A. M. Dirac, Principles of Quantum Mechanics (Oxford University Press, London, 1958)]. The theoretical search for the quantum phase operator continued after Dirac. Heitler [W. Heitler, The Quantum Theory of Radiation (Academic, London, 1954)] used Dirac's formulation in the quantization of the electromagnetic field. Perhaps Heitler's work somewhat made a landmark in the search for a quantum phase in the context of the quantum properties of the electromagnetic field and the quantum phase became a dominant research topic of quantum optics. The introduction of the photon coherent states by Glauber [R. J. Glauber, Phys. Rev. 130, 2529 (1963); 131, 2766 (1963)] and the developments in the laser physics in the 1960s enhanced the role of quantum optics in the quantumphase research. Another course was taken by Carruthers and Nieto in their seminal work [P. Carruthers and M. M. Nieto, Rev. Mod. Phys. 40, 411 (1968)], which they wisely entitled Phase and Angle Variables in Quantum Mechanics, where a revival of the canonical phase-space perspective of the quantum-phase problem was initiated. This line of canonical approach has been furthered (the author apologizes for not being able to include a complete list of important theoretical and experimental works) by the works of Rocca and Siruge [F. Rocca and M. Siruge, Commun. Math. Phys. 34, 111 (1973)], Moshinsky and Seligman [M. Moshinsky and T. H. Seligman, Ann. Phys. (N.Y.) 114, 243 (1978); J. Phys. A 12, L135 (1979)], and Newton [R. G. Newton, Ann. Phys. (N.Y.) 124, 327 (1980)]. In 1989 Pegg and Barnett introduced the most popular phase operator formalism [D. T. Pegg and S. M. Barnett, Phys. Rev. A 39, 1665 (1989)] based on a mathematical abstraction of the concept of truncated Hilbert spaces where they were able to define a Hermitian operator corresponding to a quantum phase. Although the Pegg-Barnett formalism is practical from the calculational point of view, it is not algebraic because of the arbitrary introduction of a truncation in the Hilbert space. On the opposite side, Moshinsky and Seligman's work has been recently followed by Luis and Sánchez- 
Soto [A. Luis and L. L. Sanchez-Soto, ibid. 47, 1492 (1993); 48, $752(1993)]$ to find a canonical path to the phase problem. More recently an algebraic canonical approach based on the generalized quantum-phase-space formalism was introduced by the present author [T. Hakioğlu, J. Phys. A 31, 6975 (1998); 31, 707 (1998)]. The second perspective is to understand the properties of the quantum phase by developing a theory of phase measurement. There is substantial literature on different classical and quantum measurement schemes allowing one to extract the measured properties of the phase. These schemes were mostly inherited from the recent developments in quantum optics, thus being confined to the phase properties of the electromagnetic field. In this stream, some important works are, in addition to the other references of this article, by Vogel and Schleich [W. Vogel and W. Schleich, Phys. Rev. A 44, 7642 (1991)] and Freyberger et al. [M. Freyberger, K. Vogel, and W. P. Schleich, Phys. Lett. A 176, 41 (1993)], who introduced the quantum-phase-space sampling by quadrature rotations, which was carried on successfully both experimentally and theoretically by Smithey et al. [D. T. Smithey, M. Beck, M. G. Raymer, and A. Faridani, Phys. Rev. Lett. 70, 1244 (1993); D. T. Smithey, M. Beck, J. Cooper, and M. G. Raymer, Phys. Rev. A 48, 3159 (1993)] and D'Ariano et al. [M. G. D'Ariano, C. Macchiavello, and M. G. A. Paris, ibid. 50, 4298 (1994); for a complete list see M. G. D'Ariano, in Quantum Optics and the Spectroscopy of Solids: Concepts and Advances, edited by T. Hakioğlu and A. S. Shumovsky (Kluwer Academic, Dordrecht, 1997)]. Recently, an interesting approach to the radiation phase was also made in A. Shumovsky, Opt. Commun. 136, 219 (1997); A. Shumovsky and Özgür Müstecaplioğlu, ibid. 146, 124 (1998)]. An impressive overview, from the quantum optics point of view, of the major historical developments in the 70-year-old theoretical and experimental search for the properties of the quantum phase and a substantial list of references are available in the recent books by Werner Vogel and Dirk-Gunnar Welsch, Quantum Optics (Akademia-Verlag, Berlin, 1994); L. Mandel and E. Wolf, $O p$ tical Coherence and Quantum Optics (Cambridge University Press, Cambridge, 1995). It must be stressed that these two distinct perspectives are not mutually exclusive but rather they should be considered, in the author's view, as generating complementary information on the properties of quantum phase.

[11] T. Hakioğlu, A. S. Shumovsky, and O. Aytür, Phys. Lett. A 194, 304 (1994).

[12] G. G. Stokes, Trans. Cambridge Philos. Soc. 9, 399 (1852).

[13] R. M. Azzam, Ellipsometry and Polarized Light (Elsevier, Amsterdam, 1989).

[14] W. H. McMaster, Am. J. Phys. 22, 351 (1954).

[15] J. M. Jauch and F. Rohrlich, Theory of Photons and Electrons (Springer-Verlag, Berlin, 1976).

[16] L. Mandel, Proc. Phys. Soc. London 74, 233 (1959).

[17] P. L. Kelley and W. H. Kleiner, Phys. Rev. 136, A316 (1964).

[18] R. J. Glauber, Phys. Rev. 130, 2529 (1963).

[19] L. Mandel and E. Wolf, Optical Coherence and Quantum Optics (Cambridge University Press, Cambridge, 1995).

[20] A. M. Perelomov, Generalized Coherent States and Their Applications (Springer-Verlag, Berlin, 1986); A. O. Barut and L. Girardello, Phys. Rev. A 31, 3068 (1985); 31, 3093 (1985).

[21] R. Jackiw, J. Math. Phys. 9, 339 (1968).

[22] Bernard Yurke, Samuel L. McCall, and John R. Klauder, Phys. Rev. A 33, 4033 (1986).

[23] Mark Hillery and Leonard Mlodinow, Phys. Rev. A 48, 1548 (1993).

[24] C. Brif and A. Mann, Phys. Rev. A 54, 4505 (1996).

[25] A. Luis and J. Peřina, Phys. Rev. A 53, 1886 (1996).

[26] G. M. D'Ariano and G. A. Paris, Phys. Rev. A 49, 3022 (1994).

[27] C. W. Helstrom, Quantum Detection and Estimation Theory (Academic, New York, 1976). 